\title{
Primäre und sekundäre Herkunftseffekte über den Verlauf der Sekundarstufe: Eine Dekomposition an drei Bildungsübergängen
}

\author{
Jan Scharf $(\mathbb{D} \cdot$ Michael Becker · Sophie E. Stallasch • Marko Neumann • \\ Kai Maaz
}

Eingegangen: 9. Oktober 2019 / Überarbeitet: 30. April 2020 / Angenommen: 14. Oktober 2020 / Online publiziert: 17 . November 2020

(C) Der/die Autor(en) 2020

Zusammenfassung Die Sekundarstufe ist gekennzeichnet durch eine Sequenz von Übergängen, an denen Ungleichheiten durch Leistungsdisparitäten (primäre Effekte) und Bildungsentscheidungen (sekundäre Effekte) nach sozialer Herkunft relevant werden. Diese Herkunftseffekte werden mit dem vorliegenden Beitrag erstmals anhand von Daten einer vollständigen Schülerkohorte (Hamburger KESS-Studie) über den gesamten Verlauf der Sekundarstufe vom Übergang ins Gymnasium bis zur Studienintention analysiert. Eine Dekomposition und Quantifizierung primärer und

\footnotetext{
Diese Arbeit nutzt Daten der Längsschnittstudie KESS. Der Datensatz wurde von der Freien und Hansestadt Hamburg durch die Behörde für Schule und Berufsbildung zwischen 2003 und 2012 generiert und dem Wissenschaftlichen Konsortium MILES (Methodological Issues in Longitudinal Educational Studies) für einen befristeten Zeitraum zur vertieften Bearbeitung wissenschaftlicher Fragestellungen zur Verfügung gestellt. MILES wird am Leibniz-Institut für die Pädagogik der Naturwissenschaften und Mathematik (IPN) koordiniert.
}

Zusatzmaterial online Zusätzliche Informationen sind in der Online-Version dieses Artikels (https:// doi.org/10.1007/s11618-020-00981-7) enthalten.

Dr. J. Scharf $(\bowtie) \cdot$ Prof. Dr. M. Becker · Dr. M. Neumann · Prof. Dr. K. Maaz

Abteilung Struktur und Steuerung des Bildungswesens, DIPF I Leibniz-Institut für Bildungsforschung und Bildungsinformation, Rostocker Straße 6, 60323 Frankfurt am Main, Deutschland

E-Mail: scharf@dipf.de

Prof. Dr. M. Becker

E-Mail: becker@dipf.de

Dr. M. Neumann

E-Mail: marko.neumann@dipf.de

Prof. Dr. K. Maaz

E-Mail:maaz@dipf.de

S. E. Stallasch

Lehrstuhl Quantitative Methoden in den Bildungswissenschaften, Humanwissenschaftliche Fakultät, Universität Potsdam, Karl-Liebknecht-Str. 24-25, 14476 Potsdam, Deutschland

E-Mail: stallasch@uni-potsdam.de 
sekundärer Effekte mit der KHB-Methode erfolgt dabei erstmals auch für den Eintritt in die Oberstufe. Abnehmende absolute Herkunftseffekte über den Bildungsverlauf, auf die bisherige Befunde verschiedener Stichproben verweisen, können mit dieser Studie zum Teil bestätigt werden. Zum vermuteten relativen Bedeutungszuwachs sekundärer Effekte zeigen die Ergebnisse kein eindeutiges Muster: Beim Wechsel ins Gymnasium überwiegen primäre Effekte leicht, wenn Noten als Leistungsindikator verwendet werden. Beim Eintritt in die Oberstufe bleibt die Relation nahezu unverändert. Erst bei der Studienintention fällt die relative Bedeutung sekundärer Effekte größer aus.

Schlüsselwörter Bildungsübergänge · Primäre und sekundäre Herkunftseffekte · Effektdekomposition · Vollerhebung · Soziale Ungleichheit

\title{
Primary and secondary effects of social background across secondary education: Decomposing effects at three educational transitions
}

\begin{abstract}
Secondary education is characterised by a sequence of transitions that are linked to educational inequality. Regarding social background effects, inequalities relate to disparities in educational achievement (primary effects) and educational decisions (secondary effects). In the present study, both primary and secondary effects are analysed based on an entire student cohort in the Hamburg school system (KESS study) across the course of secondary education, i.e. from entering secondary school to aiming for higher education. The KHB method is applied in order to decompose the effects, including transitions to upper secondary education. In line with previous research, total social background effects decrease across educational trajectories. No clear pattern emerges concerning an assumed increase in the relative importance of secondary effects. Primary effects operationalised by school grades are slightly predominant in the transition to lower secondary education, and nearly the same ratio is found for the transition to upper secondary education. However, regarding the aim to enter higher education, the relative importance of secondary effects is more clearly discernible.
\end{abstract}

Keywords Complete survey · Decomposition · Educational transitions · Primary and secondary effects $\cdot$ Social inequality

\section{Einleitung}

Übergänge sind ein wesentliches Merkmal gegliederter Bildungssysteme. Sie werden zugleich als eine zentrale Ursache sozialer Disparitäten in individuellen Bildungsverläufen diskutiert (Baumert et al. 2006; Becker und Lauterbach 2016; Maaz et al. 2009). Bei der Erklärung von Bildungsungleichheiten konnte dieser Zusammenhang empirisch vielfach nachgewiesen werden: Mit einer privilegierteren sozialen Herkunft ist der Besuch höher qualifizierender Schulformen wahrscheinlicher, insbesondere der Wechsel von der Grundschule auf das Gymnasium (Ditton und Krüsken 2006; Pietsch 2007). Auch bei weiteren Übergangsschwellen, wie dem Übergang in 
eine zum Abitur führende gymnasiale Oberstufe und der anschließenden Aufnahme eines Studiums, zeigen sich soziale Disparitäten (Baumert et al. 2018; Neugebauer und Schindler 2012). Bezugnehmend auf Boudons (1974) Erklärungsansatz wird dabei unterschieden, inwieweit herkunftsspezifische schulische Leistungsdisparitäten (primäre Effekte) oder aber familiäre Bildungsentscheidungen in Abhängigkeit der sozialen Herkunft (sekundäre Effekte) für das Übergangsverhalten verantwortlich sind.

Hinsichtlich der Entwicklung sozialer Disparitäten im Bildungsverlauf deuten die vorhandenen Forschungsbefunde darauf hin, dass insgesamt familiäre Einflussfaktoren über die verschiedenen Übergangsschwellen an Bedeutung verlieren und gleichzeitig sekundäre Herkunftseffekte, relativ betrachtet, an Bedeutung gewinnen (vgl. Becker 2009; Neugebauer et al. 2013). Um diese Entwicklung der absoluten und relativen Größe des Herkunftseffekts zu verdeutlichen, werden häufig der Eintritt in die weiterführende Schule und die Studienaufnahme nach dem Abitur gegenübergestellt. Weitgehend unklar ist aber weiterhin, wann und wie stark sich diese Veränderungen in der Vorhersagekraft und in der Zusammensetzung der Herkunftseffekte im Verlauf der Sekundarstufe zeigen. Für Vergleiche zwischen dem Grundschulübergang und späteren Übergangsschwellen im Bildungssystem konnten bisherige Studien zudem ausschließlich auf Stichproben selektiver Schülergruppen zurückgreifen (vgl. Watermann et al. 2014), die an den jeweiligen Übergangsschwellen im Schulsystem verblieben sind und damit gegenüber der früheren Gesamtpopulation eine - leistungsbasiert - positiv selektierte Population darstellen.

Hier setzt die vorliegende Studie an, indem sie die Analyse primärer und sekundärer Effekte über den Bildungsverlauf erstmals auf einen vollständigen Schülerjahrgang ausweitet: Primäre und sekundäre Herkunftseffekte werden auf diese Weise vergleichend an den drei Übergängen in die Sekundarstufe I, in die gymnasiale Oberstufe und in tertiäre Bildungsgänge (Studienintention) nicht nur für (selektive) Teilpopulationen wie etwa Abiturient*innen, sondern ergänzend zu bisheriger Forschung auch für die gesamte Ausgangskohorte geschätzt. Die Untersuchung eines über die gesamte Sekundarstufe empirisch begleiteten Schülerjahrgangs, der Hamburger Schülerkohorte der KESS-Studie, erlaubt es zudem, den einschlägigen Forschungsstand zur Dekomposition primärer und sekundärer Effekte für das deutsche Bildungssystem im Rahmen des institutionellen Kontexts des Schulsystems eines Bundeslandes und auf Basis der gleichen Population und gleicher Messinstrumente zu validieren. Dies bietet insbesondere den Vorteil, die für die Dekomposition des Herkunftseffekts benötigten Schulleistungen an den drei Übergangsschwellen auf gleiche Weise zu operationalisieren. Dabei dient der Leistungsstand als Mediatorvariable des Herkunftseffekts, um den indirekten (primären), über die Leistung vermittelten Effekt der sozialen Herkunft vom direkten (sekundären) Effekt zu trennen. Erstmals erfolgt dabei auch für den Übergang in die gymnasiale Oberstufe eine Quantifizierung primärer und sekundärer Herkunftseffekte. 


\section{Theoretische Rahmung und Forschungsstand: Primäre und sekundäre Herkunftseffekte im Bildungsverlauf}

Mit der Unterscheidung in primäre und sekundäre Effekte der sozialen Herkunft hat Boudon (1974) in seinem strukturell-individualistischen Ansatz dargelegt, in welcher Weise die soziale Herkunft der Familie und der Bildungserfolg des Kindes im Zusammenspiel mit den institutionellen Kontexten des Schulsystems verknüpft sind (vgl. Becker und Lauterbach 2016). Einen wesentlichen Faktor stellen die mit der sozialen Herkunft assoziierten schulischen Leistungen dar, die auf die unterschiedliche Ressourcenausstattung des Elternhauses zurückzuführen sind. So differieren etwa die elterliche Unterstützung durch unmittelbare Hilfestellung, die Schaffung einer förderlichen Lernumwelt oder kulturelle Aktivitäten und Güter, was ungleiche Startbedingungen innerhalb der Schülerschaft zur Folge hat (Maaz und Nagy 2009). Mit einer privilegierteren sozialen Herkunft fallen demnach Schulleistungen und Kompetenzen der Kinder höher aus, als es bei einer größeren „Bildungsferne“ der Familie der Fall ist (vgl. Becker 2009; Neumann et al. 2014). Da den schulischen Leistungen eine wesentliche Rolle für Übergänge im Bildungssystem zukommt, resultieren herkunftsspezifische Leistungsentwicklungen auch in unterschiedlichen Übergangsquoten an den Übergangsschwellen in der Bildungslaufbahn (primärer Effekt). Daneben wirken aber auch leistungsunabhängige, schichtspezifische familiäre Präferenzen und Kosten-Nutzen-Abwägungen bezüglich der einzuschlagenden Bildungswege (sekundärer Effekt) auf soziale Ungleichheiten in den Bildungswegen. Verdeutlicht am Beispiel unterer Sozialschichten bedeutet dies, dass die Kosten weiterführender Bildung (Abitur) als größer wahrgenommen werden und deren Nutzen geringer bewertet wird, wenn diese nicht mit der eigenen sozialen Position korrespondiert und für das Bildungsziel nicht als notwendig erachtet wird (Dumont et al. 2014, S. 144).

Der bildungssoziologische Ansatz von Boudon (1974) wurde in rationalen Entscheidungsmodellen vertieft (vgl. exemplarisch Esser (1999) anhand der Wert-Erwartungstheorie) und unter anderem für den deutschen Kontext empirisch geprüft (Becker 2003; Stocké 2007a), wobei sich neben primären auch sekundäre Effekte als relevant erwiesen. Letztere werden besonders kritisch diskutiert, da sie meritokratischen Leistungsprinzipien widersprechen (Becker 2009).

Im Folgenden sollen die zentralen Forschungsbefunde für die drei im vorliegenden Beitrag untersuchten Übergangsschwellen dargestellt werden. Vor dem Hintergrund der zum Teil uneinheitlichen Befundlage soll in diesem Zusammenhang auch auf methodische Implikationen der Identifikation primärer und sekundärer Herkunftseffekte eingegangen werden.

\subsection{Die erste Übergangsschwelle: Übergang von der Grundschule in die Sekundarstufe}

An der ersten hier zu untersuchenden Übergangsschwelle im deutschen Bildungssystem von der Grundschule in die weiterführende Schule wirken verschiedene Faktoren auf das Übergangsverhalten der Schüler*innen. In ihrer Konzeptualisierung zur Ermittlung und Quantifizierung primärer und sekundärer Herkunftseffekte haben Maaz 
und Nagy (2009) diesbezüglich eine Erweiterung des Ansatzes von Boudon (1974) vorgenommen. Sie unterscheiden zum einen primäre und sekundäre Effekte auf die Schülerbeurteilung (zunächst auf die Schulnoten, dann auf die Laufbahnempfehlung) und zum anderen auf das konkrete Übergangsverhalten in Form des Gymnasialbesuchs - mit dem Ziel ,einer chronologischen Perspektive“ (Maaz und Nagy 2009, S. 158) auf Herkunftseffekte. Das Konzept konnten sie anhand bundesweit repräsentativer Erhebungsdaten aus der 4. Jahrgangsstufe (TIMSS-ÜBERGANG-Studie) in einer pfadanalytischen Zerlegung des sozialen Herkunftseffekts, operationalisiert über den sozioökonomische Status der Eltern (Highest International Socio-Economic Index of Occupational Status, HISEI; Ganzeboom et al. 1992), prüfen. Primäre Effekte der sozialen Herkunft, vermittelt über Testleistungen der Schüler*innen, überwogen in der Notengebung, während primäre und sekundäre Effekte bei der Laufbahnempfehlung in etwa gleich groß ausfielen (die Angaben zu Noten und Laufbahnempfehlungen basierten auf Auskünften der Schulen). Für den im Elternfragebogen erhobenen realisierten Übergang auf das Gymnasium zeigte sich, dass der Gesamteffekt der sozialen Herkunft zu $41 \%$ auf primäre Effekte und zu $59 \%$ auf sekundäre Effekte zurückzuführen war. Für letztere konnte im Modell von Maaz und Nagy (2009, S. 173) ein indirekter Effekt mit einem Anteil von 54\% ermittelt werden, der besagt, dass etwas mehr als die Hälfte des sekundären Effekts am Übergang nach der Grundschule auf zuvor auftretende sekundäre Effekte in der Notenvergabe und Übergangsempfehlung zurückzuführen war. Darin scheinen sich etwa spezifische Beurteilungen (Maaz und Nagy 2009), Erfolgserwartungen (Neugebauer 2010, S. 204) sowie die eingeschätzte elterliche Unterstützung (Ditton und Krüsken 2006, S. 368) der Lehrkräfte hinsichtlich des familiären Hintergrunds der Schüler*innen widerzuspiegeln, die in der jüngeren Forschungsliteratur mitunter auch als tertiäre Effekte bezeichnet werden (vgl. Esser 2016, S. 341; siehe für einen Überblick Helbig und Morar 2017).

Die identische Relation primärer und sekundärer Herkunftseffekte (41 zu 59\%), operationalisiert über den familiären Bildungshintergrund in Form des höchsten elterlichen Schulabschlusses, fand sich auch bei Neugebauer (2010) mit bundesweiten Daten des DJI-Kinderpanels. Unter Verwendung von Schulnoten als Indikator primärer Herkunftseffekte wurden in dieser Studie tatsächliche und kontrafaktische Übergangsraten auf das Gymnasium (odds ratio) mit der Dekompositionsmethode nach Erikson et al. (2005) berechnet. Hingegen zeigte sich bei gleicher Methode unter Verwendung der IGLU-E 2001 Testleistungen (Neugebauer et al. 2013), dass der primäre Effekt (operationalisiert über Schulnoten) sowohl für den Bildungshintergrund (im Mittel 56\%) als auch für die soziale Herkunftsklasse (60\%) überwog. Zudem konnte Neugebauer (2010) über einen Vergleich der Bundesländer hinsichtlich der institutionellen Übergangsregularien aufzeigen, dass die sekundären Effekte ein geringeres relatives Gewicht aufweisen, wenn die Übergangsempfehlung verbindlich ist (siehe auch Gresch et al. 2010).

Vor diesem Hintergrund betrifft eine erste Implikation aus dem Forschungsstand die Operationalisierung primärer Effekte. Mit der Wahl der Leistungsvariable sind letztlich konzeptionelle Annahmen verknüpft: In den meisten Forschungsarbeiten werden die Schulnoten verwendet. Ein wesentliches Argument ist, dass Schulnoten gegenüber in Forschungsstudien erfassten Testleistungen eine größere Entschei- 
dungsrelevanz für Eltern haben (vgl. Neugebauer et al. 2013, S. 66). Wie Maaz und Nagy (2009) in ihrem Modell dargelegt haben, wirken jedoch bereits sekundäre Effekte auf die Leistungsbeurteilung (Notengebung) der Schüler*innen. Dem ließe sich durch die Verwendung von standardisierten und sozial unverzerrten Testleistungen, welche die unmittelbaren, im Zuge der empirischen Datenerhebung gewonnenen Lernstände in verschiedenen Kompetenzbereichen widerspiegeln, zumindest in Teilen entgegenwirken, um so einer möglichen Überschätzung primärer Effekte vorzubeugen (vgl. etwa Müller-Benedict 2007; Stocké 2007b).

\subsection{Die zweite Übergangsschwelle: Übergang in die gymnasiale Oberstufe}

Der Wechsel in die gymnasiale Oberstufe, v. a. nach Erreichen des mittleren Schulabschlusses an nichtgymnasialen Schulformen als weitere, alternative Option neben dem quasi standardmäßigen Übergang in die Oberstufe am Gymnasium, wird als zweite wichtige Übergangsschwelle fokussiert. In einer der wenigen Untersuchungen für diesen Übergang konnten Trautwein et al. (2011) mit Daten aus der 10. Jahrgangsstufe (TOSCA-10) baden-württembergische Schüler*innen zum Ende der Sekundarstufe I in den beiden Schulformen Gymnasium und Realschule vergleichen. Dabei untersuchten sie die Frage, inwieweit die Öffnung der Bildungswege soziale Disparitäten in den Bildungsverläufen und im Bildungserwerb mindern kann, bzw. ob umgekehrt die zusätzlichen Opportunitäten im Bildungssystem vermehrt von privilegierten Herkunftsschichten genutzt und damit Ungleichheiten verstärkt werden (vgl. hierzu auch Hillmert und Jacob 2005). Für das Land Baden-Württemberg mit seinem stark ausgebauten Bereich beruflicher Gymnasien zeigten die Untersuchungsergebnisse, dass Schüler*innen, die von der Realschule in die gymnasiale Oberstufe wechselten (in der Regel an ein berufliches Gymnasium), im Mittel einen geringeren Bildungshintergrund aufwiesen als diejenigen, die bereits auf dem Gymnasium waren. Zugleich aber unterschieden sich auch diejenigen Realschüler*innen, die von ihrer erworbenen Übergangsberechtigung für die gymnasiale Oberstufe Gebrauch machten, von den Übrigen durch eine höhere soziale Herkunft (HISEI). Trotz der Etablierung beruflicher Gymnasien als alternativem Hochschulzugangsweg in Baden-Württemberg konnten demnach auch an dieser Übergangsschwelle ,soziale Selektionsprozesse“ (Maaz 2006, S. 233) identifiziert werden. Eine Effektzerlegung in primäre und sekundäre Herkunftsanteile wurde nicht vorgenommen.

Im Rahmen der BERLIN-Studie zur Evaluation der Umstellung auf das zweigliedrige Schulsystem im Land Berlin haben Baumert et al. (2018) gezeigt, dass sich ein geringerer sozioökonomischer Status der Familie negativ auf die Chancen auswirkt, an nichtgymnasialen Schulen eine Berechtigung zum Übergang in die gymnasiale Oberstufe zu erhalten (vgl. auch Maaz et al. 2017). Diese Befunde ließen sich selbst dann bestätigen, wenn „Fachleistungen, die durch objektive Tests erfassbar sind, kognitive Grundfähigkeiten, die Schulnoten in den Kernfächern, Lernmotivation, Schulverhalten sowie schulrelevante Persönlichkeitsmerkmale“ (Baumert et al. 2018, S. 620) simultan kontrolliert wurden. Inwieweit diese Befunde auch auf den realisierten Übergang übertragbar sind, wurde bislang nicht untersucht.

$\mathrm{Zu}$ Ungleichheiten beim Übergangsverhalten in die gymnasiale Oberstufe wird ein grundsätzlicher Forschungsbedarf identifiziert (Baumert et al. 2018, S. 595). Zu- 
sätzlich stellt sich für den vorliegenden Beitrag eine zweite wesentliche Implikation heraus: Unseres Wissens nach liegen bislang keine Studien vor, die für diese Übergangschwelle im deutschen Kontext explizit eine Quantifizierung und Dekomposition primärer und sekundärer Effekte vornehmen. Entsprechende Analysen können allerdings Aufschluss darüber geben, ob sich bereits an dieser Übergangsschwelle die relative Bedeutung primärer und sekundärer Effekte zwischen Grundschulübergang und dem Übergang ins Studium verschiebt.

\subsection{Die dritte Übergangsschwelle (Übergang in die Hochschule) und eine Gesamtbetrachtung}

Auch an der dritten und letzten Übergangsschwelle im Schulsystem, dem Übergang in die Hochschule im Anschluss an den Erwerb des Abiturs, werden soziale Herkunftseffekte relevant. Wie Watermann et al. (2014) in ihrem Forschungsüberblick dokumentieren, fallen die Herkunftseffekte an dieser späten Stelle des Bildungssystems dem Transitionsmodell von Mare (1980) folgend jedoch insgesamt geringer aus, da im Zuge der Sequenz von Bildungsentscheidungen an den Übergangsschwellen mit jeder höheren Bildungsstufe bereits eine durch herkunftsspezifische Entscheidungsmuster und Leistungen selektierte Schülerpopulation die Bildungsangebote nutzt. Dies hat zur Folge, dass die Schülerschaft in sozialer und leistungsbezogener Hinsicht homogener wird (Hillmert und Jacob 2005), wodurch die Vorhersagekraft der sozialen Herkunft für getroffene Übergangsentscheidungen insgesamt abnimmt. Während gemäß der Selektionshypothese wie beschrieben also insbesondere die Selbst- und Fremdselektion der Schülerpopulation die wesentliche theoretische Argumentationsgrundlage für die abnehmenden Herkunftseffekte darstellt, betont die Lebensverlaufshypothese, dass die Schüler*innen an den aufeinanderfolgenden Bildungsübergängen zunehmend unabhängiger von ihrer Herkunftsfamilie werden und der familiäre Einfluss auf das Entscheidungsverhalten der Schüler*innen in der Folge ebenfalls abnimmt (Blossfeld und Shavit 1993, S. 34f.).

Trotz der durch die empirische Befundlage indizierten generellen Abnahme sozialer Herkunftseffekte im Bildungsverlauf legen die Befunde zur Frage der relativen Größe der primären und sekundären Herkunftseffekte beim Übergang ins Studium einen deutlich größeren Anteil sekundärer Effekte nahe (Watermann et al. 2014). Becker (2009) konnte mit Daten sächsischer Abiturient*innen in einer Simulationsstudie zeigen, dass die sekundären Effekte (soziale Schicht) für eine Studienintention mit $63 \%$ gegenüber den anhand der (selbst eingeschätzten) Schulleistung gemessenen primären Effekten größer ausfielen. Maaz (2006) wies mit TOSCA-Daten in allgemeinbildenden und beruflichen Gymnasien in Baden-Württemberg neben den mit Hilfe von Testleistungen und Schulnoten operationalisierten primären Effekten auch sekundäre Effekte auf die Studienintention sowie auf den realisierten Hochschulübergang nach, wobei keine explizite Quantifizierung der relativen primären und sekundären Herkunftsanteile erfolgte. Untersuchungen für die tatsächliche Aufnahme eines Hochschulstudiums auf der Datengrundlage des DZHW-Studienberechtigtenpanels zeigten, dass sekundäre Effekte teilweise deutlich überwogen und über Kohorten hinweg sogar an Bedeutung gewonnen haben (Schindler und Lörz 2012). Für die soziale Klassenzugehörigkeit kommen Schindler und Reimer (2010) 
auf rund $80 \%$ sekundären Herkunftsanteil. Neugebauer et al. (2013) weisen sekundäre Effekte des Bildungshintergrunds von $72 \%$ (und der sozialen Herkunftsklasse von $53 \%$ ) nach.

Da bislang keine Gesamtbetrachtung der Herkunftseffekte an den aufeinanderfolgenden Übergangsschwellen im deutschen Schulsystem anhand einer Schülerkohorte vorliegt, wird die dritte und für die vorliegende Studie entscheidende Implikation ersichtlich: So beruhen die hier skizzierten Studien, die Herkunftseffekte an zwei oder mehr Übergängen analysieren, für jeden Übergang auf unterschiedlichen Datensätzen (siehe Becker 2009; Neugebauer et al. 2013). Um die jeweilige Bedeutung primärer und sekundärer Herkunftseffekte im Verlauf nachzuzeichnen, mussten insofern Ergebnisse verschiedener Stichproben (und Bundesländer) miteinander verglichen werden. Zudem wurden insbesondere an den späteren Übergangsschwellen bereits stark vorselektierte und im Vergleich zur ursprünglichen Ausgangskohorte der Schüler*innen beim Eintritt in das Schulsystem vergleichsweise homogene Schülergruppen zugrunde gelegt (vgl. Mare 1980), etwa Abiturient*innen für den Übergang in die Hochschule. Aussagen über die Kumulation von Herkunftseffekten in der Gesamtkohorte und darauf bezogene vergleichende Betrachtungen primärer und sekundärer Herkunftseffekte über die verschiedenen Übergangsschwellen hinweg sind damit nur eingeschränkt möglich.

\section{Die vorliegende Studie}

Ziel der vorliegenden Untersuchung ist eine Dekomposition primärer und sekundärer Herkunftseffekte an den entscheidenden Übergangsschwellen über den Verlauf der Sekundarstufe, d.h. am Übergang von der Grundschule in die Sekundarstufe, von der Sekundarstufe I in die gymnasiale Oberstufe sowie am (intendierten) Übergang in ein Studium. Auf Grundlage einer konstanten und kompletten Schülerkohorte innerhalb eines Bundeslandes soll damit zum einen der bisherige Kenntnisstand validiert werden, zum anderen werden die oben identifizierten Implikationen und Desiderata aufgegriffen: Zusammenfassend bedeutet dies, dass erstens Noten und standardisierte Tests als Leistungsindikatoren für die Quantifizierung primärer Herkunftseffekte gegenübergestellt werden, zweitens die Übergangsschwelle zur Oberstufe erstmals integriert wird und drittens jeweils der vollständige Schülerjahrgang an den drei betrachteten Übergangsschwellen einbezogen und somit die Dekomposition über die verschiedenen Übergangsschwellen mit Blick auf die zugrundeliegende Schülerpopulation vergleichbar gemacht wird. Darüber hinaus werden in der Literatur gängige, aber zwischen Studien bzw. Stichproben lediglich eingeschränkt vergleichbare Operationalisierungen der zentralen Indikatoren (Schulleistungen, soziale Herkunft) über die Übergangsschwellen hinweg konstant gehalten. Im Folgenden stellen wir die konkreten Fragestellungen und Forschungshypothesen unserer Untersuchung dar.

In einem ersten Schritt wird untersucht, zu welchen relativen Anteilen sich primäre und sekundäre Effekte der sozialen Herkunft an den beschriebenen Übergangsschwellen zusammensetzen und ob sich das relative Gewicht je nach verwendetem Leistungs- und Herkunftsindikator unterscheidet. In Abb. 1 wird die Zerlegung des totalen Herkunftseffekts auf den Bildungsübergang in die Pfade des direkten und des 
Abb. 1 Direkte und indirekte Pfade in den Modellen und abgeleitete Hypothesen

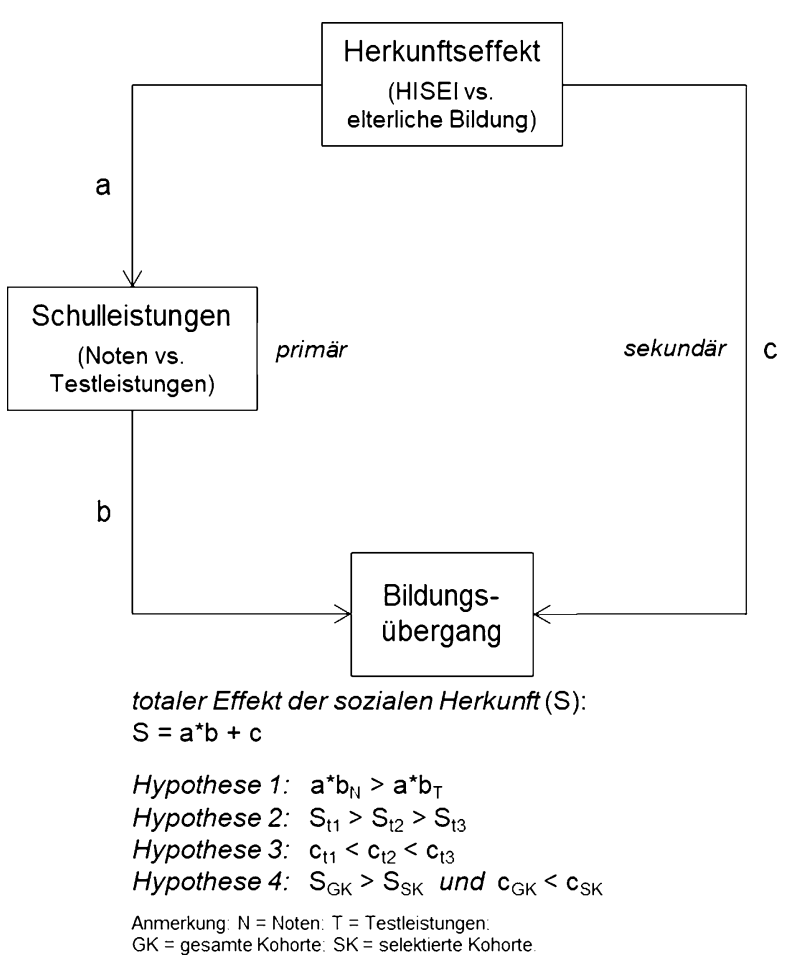

indirekten Effekts veranschaulicht. Mit dem direkten Effekt (c) werden sekundäre Herkunftseffekte nach Kontrolle der Leistungsindikatoren geschätzt. Der primäre Herkunftseffekt ergibt sich aus dem Produkt des Effekts der sozialen Herkunft auf die Schulleistungen (a) und des Effekts der Leistungen auf den Bildungsübergang (b) (=indirekter Effekt $\mathrm{a} * \mathrm{~b})$. Hinsichtlich der ersten im Forschungsstand identifizierten Implikation wird dabei angenommen (vgl. Stocké 2007b; Neugebauer et al. 2013), dass primäre Herkunftseffekte überschätzt werden, wenn die Schulleistung über Schulnoten $(N)$ anstelle von Testleistungen $(\mathrm{T})$ operationalisiert wird $\left(\mathrm{a} * \mathrm{~b}_{\mathrm{N}}>\mathrm{a} *\right.$ $\mathrm{b}_{\mathrm{T}}$ ). Sekundäre Effekte sollten demnach stärker ausfallen, wenn standardisierte Testleistungen als (gegenüber Noten) objektivere Leistungsindikatoren herangezogen werden (Hypothese 1). Zweitens wird danach gefragt, wie groß die Herkunftseffekte insgesamt auf die Übergangswahrscheinlichkeiten an den drei Übergangsschwellen ausfallen. Aufgrund der Selektion der Schülerschaft einerseits (Mare 1980) und zunehmender Unabhängigkeit vom Elternhaus im Lebensverlauf andererseits (Blossfeld und Shavit 1993; vgl. Abschn. 2.3) wird von einer abnehmenden Erklärungskraft familiärer sozialer Einflussfaktoren an den späteren Übergangsschwellen (Übergang in die gymnasiale Oberstufe und insbesondere Übergang ins Studium) ausgegangen (Hypothese 2: $\mathrm{S}_{\mathrm{t} 1}>\mathrm{S}_{\mathrm{t} 2}>\mathrm{S}_{\mathrm{t} 3}$ ). Es schließt sich drittens die Frage an, ob bzw. an welcher Stelle und wie stark im Verlauf der Sekundarstufe ein relativer Bedeutungszuwachs des sekundären Effekts zu verzeichnen ist. Unter Bezugnahme auf den bisherigen Forschungsstand (vgl. Becker 2009) erwarten wir mit jeder weiteren Übergangsschwelle eine zunehmende Bedeutung sekundärer Effekte $\left(c_{t_{1}}<c_{t 2}<c_{t 3}\right)$ 
sowie entsprechend eine rückläufige Bedeutung primärer Effekte (Hypothese 3). Für den Übergang in die gymnasiale Oberstufe $\left(c_{\mathrm{t} 2}\right)$ ist dabei offen, ob die Relation primärer und sekundärer Effekte eher der Relation am Grundschulübergang $\left(c_{t 1}\right)$ oder am Studienübergang $\left(c_{t 3}\right)$ ähnelt. Darüber hinaus werden Selektions- und potentielle Lebensverlaufseffekte voneinander getrennt, um Effektmuster, die vorrangig auf der zunehmenden Homogenisierung der Schülerschaft im Zuge der Selektionsprozesse beruhen, von den Effektmustern in der Gesamtkohorte zu unterscheiden: Für das Ergebnismuster des dritten Übergangs ins Studium sollte es von wesentlicher Bedeutung sein, ob die gesamte Kohorte (GK) oder lediglich die bereits vorselektierte Kohorte (SK), wie in der bisherigen Literatur gängig, zugrunde gelegt wird. Es ist zu erwarten, dass einerseits die absoluten Effekte größer ausfallen $\left(S_{\mathrm{GK}}>\mathrm{S}_{\mathrm{SK}}\right)$ und andererseits die sekundären Effekte ein geringeres relatives Gewicht erhalten $\left(\mathrm{c}_{\mathrm{GK}}<\mathrm{c}_{\mathrm{SK}}\right)$, wenn anstelle der selektiven Übergangsgruppe die Schüler*innen der gesamten Ausgangskohorte betrachtet werden (Hypothese 4).

\section{Methoden}

\subsection{Datengrundlage}

Die Datenbasis der Analysen bildet die Schulleistungsstudie Kompetenzen und Einstellungen von Schülerinnen und Schülern (KESS), mit der im Auftrag der Behörde für Schule und Berufsbildung der Freien und Hansestadt Hamburg die Lernstände aller Schüler*innen einer Kohorte im allgemeinbildenden Schulsystem im Längsschnitt erfasst wurden. Ausgehend von der ersten Erhebung im Juni 2003 zum Ende des vierten Schuljahres (KESS 4), wurde diese Schülerkohorte unter anderem beim Übergang von der Sekundarstufe I in die Sekundarstufe II (KESS 10/11) und am Ende der gymnasialen Oberstufe (KESS 12/13) in einer Vollerhebung getestet und befragt (Bos und Pietsch 2006; Vieluf et al. 2011, 2014).

In Hamburg besuchen alle Kinder die vierjährige Grundschule, die zum Ende des ersten Halbjahres der 4. Klasse eine Empfehlung für die weiterführende Schulform ausspricht. Da der Stadtstaat Hamburg zu den Bundesländern mit nicht bindender Schullaufbahnempfehlung zählt (Gresch et al. 2010, S. 214), oblag die Entscheidung für die Schulform der zum damaligen Zeitpunkt viergliedrigen Sekundarstufe I den Eltern und Kindern der befragten Kohorte: Die Schüler*innen wechselten in Hauptund Realschule, Gymnasium oder eine Kooperative oder Integrierte Gesamtschule (vgl. Lehberger und de Lorent 2012). Nach zweijähriger Beobachtungsstufe entschieden Haupt- und Realschulen, Gymnasien und Kooperative Gesamtschulen auf Grundlage der erbrachten Leistungen der Schüler*innen über die zu besuchende Schulform ab der 7. Jahrgangsstufe. ${ }^{1}$ Da das Abitur an grundständigen Gymnasien

\footnotetext{
1 Bezüglich dieser Besonderheit des Hamburger Schulsystems konnte Stubbe (2009) für die KESS-Kohorte ermitteln, dass der Großteil der Schulformwechsel auf der notwendigen Entscheidung über die Schulform an Haupt- und Realschulen beruht (bei zugleich geringen Herkunftseffekten) und an Gymnasien vor allem Abgänge zu beobachten waren. Für den Fokus der vorliegenden Studie, der auf der Fortsetzung der Bildungslaufbahn auf der nächsthöheren Bildungsstufe liegt, wird diese Übergangsschwelle damit als nachrangig erachtet und analytisch nicht weiter verfolgt.
} 
bereits nach acht Jahren erworben wird, hat das 10. Schuljahr an Hamburger Gymnasien als letztem Jahr der Sekundarstufe I sowie erstem Jahr der Oberstufe eine „Gelenkfunktion“ (Lehberger und de Lorent 2012, S. 78). Zum Erhebungszeitpunkt KESS 10/11 wurden am Ende des Schuljahres 2008/2009 alle Zehntklässler*innen an Realschulen, Integrierten Haupt- und Realschulen und Gesamtschulen befragt und getestet, am Beginn des darauf folgenden Schuljahres (2009/2010) in Klasse 11 alle Schüler*innen an Gymnasien sowie in der Vorstufe (Einführungsphase der Oberstufe) der Aufbaugymnasien an Gymnasien und der Vorstufe der Beruflichen Gymnasien (Vieluf et al. 2011). An der abschließenden Erhebung nahmen 2011 - im Zeitraum zwischen den schriftlichen und mündlichen Abiturprüfungen - 82\% der Abiturient*innen an grundständigen Gymnasien (KESS 12) und 2012 bei einer Teilnahmequote von $92 \%$ die Abiturient*innen aus der dreijährigen gymnasialen Oberstufe (KESS 13) teil (Vieluf et al. 2014, S. 4).

In den nachfolgenden Analysen wurden aus den jeweiligen (Voll-)Erhebungen 13.873 Schüler*innen zu KESS 4 einbezogen, 12.551 Schüler*innen zu KESS 10/11 (davon $N_{\text {KESS } 10}=6865, N_{\text {KESS }}{ }_{11}=5686$ ) und 6632 Schüler*innen zu KESS 12/13 (davon $N_{\text {KESS }} 12=3822, N_{\text {KESS }}{ }_{13}=2810$ ). Ausgeschlossen wurden für alle Wellen Schüler*innen, die eine Förderschule besuchten $\left(N_{\text {KESS }} 4=141, N_{\text {KESS } 10 / 11}=13\right)$, für KESS 10/11 diejenigen Neuzugänge, für die die Zuordnung zu einer Schulform in der Klassenstufe vor dem Übertritt nicht möglich war (z. B. aus anderen Bundesländern zugezogen; $N=759$ ), sowie ebenfalls in KESS 10/11 Schüler*innen ohne valide Klassen-ID $(N=5)$. Im Zuge der Befragungen konnten zudem designbedingt Gymnasiast*innen, die entweder nach der 10. Jahrgangsstufe die Schule verlassen haben oder das 10. Schuljahr wiederholt haben, nicht einbezogen werden.

\subsection{Instrumente}

Übergänge Für den Übergang nach der Grundschule dient der Besuch des Gymnasiums als akademisch anspruchsvollste Schulform als abhängige Variable. Die Information über die voraussichtlich besuchte Schulform in Klasse 5 wurde dem Elternfragebogen aus KESS 4 entnommen. Es wird davon ausgegangen, dass diese Angaben den tatsächlich realisierten Übergang sehr gut approximieren, da die Erhebung für KESS 4 nach bereits erfolgten Anmeldungen an den weiterführenden Schulen durchgeführt wurde. ${ }^{2}$ Für den Übergang in die gymnasiale Oberstufe nach der Sekundarstufe I wurde die Angabe über den Besuch der gymnasialen Oberstufe in Klasse 11 aus der Schülerteilnahmeliste in KESS 11 als abhängige Variable herangezogen. Vor dem Übergang nach der Sekundarstufe II wurde die Studienintention über eine Frage zur Absicht einer Studienaufnahme aus den in KESS 12 und KESS 13 eingesetzten Schülerfragebögen operationalisiert (5-stufige Likert-Skala mit 1=,auf keinen Fall“ bis 5=,,auf jeden Fall“). Diese Angaben wurden für die Analysen dichotomisiert, indem die beiden höchsten Kategorien ( $1=$, auf jeden Fall/ wahrscheinlich“) wie in entsprechenden Studien üblich (vgl. Maaz 2006; Schneider

\footnotetext{
${ }^{2}$ Um die vorliegenden Informationen voll auszuschöpfen, wurden die Angaben aus KESS 4 mit den retrospektiven Angaben zur besuchten Schulform in Klasse 5 aus der Schülerteilnahmeliste in KESS 7 (Datenerhebung zu Beginn des 7. Schuljahres) aufgefüllt.
} 
et al. 2017) als bestehende Studienintention gewertet und den untersten drei Kategorien $(0=$, ,eventuell/unwahrscheinlich/auf keinen Fall“") gegenübergestellt wurden.

Schulleistungen Hinsichtlich der schulischen Leistungen liegen einerseits standardisierte Testleitungen vor, andererseits Schulnoten der Schüler*innen. Aus KESS 4 und KESS 10/11 wurden die erfassten Testleistungen in den Domänen Mathematik und Leseverständnis und aus KESS 10/11 in Englisch einbezogen (sowie für zusätzliche Analysen zu KESS 4 in Sachunterricht), aus KESS 12/13 Leistungstests in den Domänen Mathematik und Englisch (und zusätzlich Naturwissenschaften). Für alle Leistungstests wurde eine eindimensionale Rasch-Skalierung vorgenommen (vgl. Vieluf et al. 2011). Die Reliabilitäten für Mathematik liegen bei $R_{\text {wle }}=0,73 \mathrm{zu}$ $\operatorname{KESS~4,~} \mathrm{R}_{\mathrm{wle}}=0,85 \mathrm{zu}$ KESS 10/11 und $\mathrm{R}_{\mathrm{wle}}=0,75 \mathrm{zu}$ KESS 12/13, für das Leseverständnis bei $\mathrm{R}_{\mathrm{wle}}=0,82$ zu KESS 4 und KESS 10/11, für Englisch (C-Test) bei $\mathrm{R}_{\mathrm{wle}}=0,95 \mathrm{zu}$ KESS $10 / 11$ und bei $\mathrm{R}_{\mathrm{wle}}=0,82 \mathrm{zu}$ KESS 12/13 sowie für Sachunterricht (KESS 4) bei $R_{\text {wle }}=0,58$ und für Naturwissenschaften (KESS 12/13) bei $R_{\text {wle }}=0,52$ (Feddermann et al. 2019). Als weiteres Maß für die Schulleistung wurden für alle Erhebungswellen die Schulnoten in den Fächern Mathematik und Deutsch verwendet sowie zusätzlich zu KESS 4 die Schulnoten in Sachunterricht, zu KESS 10/11 in Englisch und zu KESS 12/13 in Englisch und Naturwissenschaften. Die Angaben zu den Schulnoten entstammen den Schülerteilnahmelisten und beziehen sich jeweils auf die Jahrgangsstufe vor dem Übergang (vgl. Tab. 1 zu deskriptiven Angaben).

Soziale Herkunft Um den Vergleich unterschiedlicher gängiger Operationalisierungen der sozialen Herkunft zu ermöglichen, werden in der vorliegenden Studie zwei Herkunftsmaße verwendet: Einerseits wird als Maß für den sozioökonomischen Status der Herkunftsfamilie der höchste International Socio-Economic Index of Occupational Status (HISEI; Ganzeboom et al. 1992) in der Familie herangezogen. Andererseits wird der Bildungshintergrund der Familie über den höchsten erreichten Schulabschluss der Eltern in dichotomisierter Form operationalisiert $(0=$ keine (Fach-)Hochschulreife, 1 = mindestens ein Elternteil mit (Fach-)Hochschulreife).

Tab. 1 gibt einen Überblick zu den Übergangsquoten an den drei Übergangsschwellen und den unabhängigen Analysevariablen. An der ersten Übergangsschwelle wechselten 43,4\% der Grundschüler*innen auf das Gymnasium. In die gymnasiale Oberstufe gingen an der zweiten Übergangsschwelle 67,0\% der Schüler*innen über. Eine Studienintention äußerten zum Ende der Sekundarstufe II 72,1\% der befragten Schülerinnen und Schüler der gymnasialen Oberstufe. Die Intention, ein Studium zu beginnen, entspricht damit annähernd der Brutto-Studierquote (Studium aufgenommen oder sicher geplant) des Abschlussjahrgangs 2012, die nach Angaben des DZHW für Studienberechtigte mit allgemeiner Hochschulreife bei $80 \%$ lag (Deutschland) und in Hamburg für alle Studienberechtigten bei $78 \%$ (vgl. Schneider et al. 2017). 
Tab. 1 Übergangsquoten an den drei Übergangsschwellen und deskriptive Angaben zu den unabhängigen Analysevariablen (beobachtete Daten)

\begin{tabular}{|c|c|c|c|c|c|}
\hline & $N_{\text {total }}$ & $N_{\mathrm{ja}}$ & $\%$ & $M$ & $S D$ \\
\hline \multicolumn{6}{|l|}{ KESS 4} \\
\hline Übergang auf das Gymnasium & 12.642 & 5482 & 43,4 & - & - \\
\hline $\begin{array}{l}\text { Bildungshintergrund (mindestens ein Eltern- } \\
\text { teil mit (Fach-)Hochschulreife) }\end{array}$ & 10.991 & 5803 & 52,8 & - & - \\
\hline HISEI & 9715 & - & - & 50,42 & 16,75 \\
\hline Mathematiknote $^{\mathrm{a}}$ & 10.499 & - & - & 4,15 & 1,02 \\
\hline Deutschnote $\mathrm{a}^{\mathrm{a}}$ & 10.495 & - & - & 4,23 & 0,83 \\
\hline Sachunterrichtnote $\mathrm{a}^{\mathrm{a}}$ & 10.492 & - & - & 4,32 & 0,92 \\
\hline Testleistung in Mathematik & 13.295 & - & - & 100,44 & 29,88 \\
\hline Testleistung im Leseverständnis & 13.292 & - & - & 100,45 & 29,79 \\
\hline Testleistung in Sachunterricht & 6645 & - & - & 100,45 & 29,81 \\
\hline \multicolumn{6}{|l|}{ KESS 10/11 } \\
\hline Übergang in die gymnasiale Oberstufe & 12.551 & 8411 & 67,0 & - & - \\
\hline $\begin{array}{l}\text { Bildungshintergrund (mindestens ein Eltern- } \\
\text { teil mit (Fach-)Hochschulreife) }\end{array}$ & 9370 & 5569 & 59,4 & - & - \\
\hline HISEI & 7726 & - & - & 53,05 & 16,37 \\
\hline Mathematiknote $^{\mathrm{a}}$ & 7759 & - & - & 5,77 & 1,59 \\
\hline Deutschnote ${ }^{\mathrm{a}}$ & 7765 & - & - & 6,02 & 1,40 \\
\hline Englischnote $^{\mathrm{a}}$ & 7734 & - & - & 5,94 & 1,46 \\
\hline Testleistung in Mathematik & 10.181 & - & - & 156,59 & 21,94 \\
\hline Testleistung im Leseverständnis & 9969 & - & - & 156,47 & 23,73 \\
\hline Testleistung in Englisch & 3806 & - & - & 143,81 & 29,02 \\
\hline \multicolumn{6}{|l|}{ KESS 12/13 } \\
\hline Studienintention: & 4983 & - & - & - & - \\
\hline Auf keinen Fall & - & 220 & 5,0 & - & - \\
\hline Wahrscheinlich nicht & - & 339 & 6,8 & - & - \\
\hline Eventuell & - & 830 & 16,7 & - & - \\
\hline Wahrscheinlich & - & 1505 & 30,2 & - & - \\
\hline Auf jeden Fall & - & 2089 & 41,9 & - & - \\
\hline $\begin{array}{l}\text { Bildungshintergrund (mindestens ein Eltern- } \\
\text { teil mit (Fach-)Hochschulreife) }\end{array}$ & 6171 & 4274 & 69,3 & - & - \\
\hline HISEI & 3743 & - & - & 56,97 & 16,08 \\
\hline Mathematiknote $^{\mathrm{a}}$ & 6032 & - & - & 4,16 & 1,15 \\
\hline Deutschnote $^{\mathrm{a}}$ & 5521 & - & - & 3,73 & 1,02 \\
\hline Englischnote $^{\mathrm{a}}$ & 5478 & - & - & 4,24 & 0,99 \\
\hline Naturwissenschaftennote ${ }^{\mathrm{a}}$ & 6010 & - & - & 3,81 & 1,11 \\
\hline Testleistung in Mathematik & 6259 & - & - & 479,65 & 87,58 \\
\hline Testleistung in Englisch & 5725 & - & - & 163,93 & 17,37 \\
\hline Testleistung in Naturwissenschaften & 2937 & - & - & 172,81 & 32,92 \\
\hline
\end{tabular}

$N(\operatorname{KESS} 4)=13.873 ; N(\operatorname{KESS} 10 / 11)=12.551 ; N(\operatorname{KESS} 12 / 13)=6632$

aAlle Schulnoten wurden umgepolt, sodass eine höhere Ausprägung für einen besseren Notendurchschnitt steht. Aufgrund unterschiedlicher Notensysteme wurden die Schulnoten zu KESS 10/11 in eine schulformübergreifende Metrik mit Werten zwischen 1 und 9 gebracht, um den Notendurchschnitt zwischen den Schulformen vergleichbarer zu machen 


\subsection{Datenanalyse}

Um die Herkunftseffekte im Hinblick auf die Anteile primärer und sekundärer Effekte zu zerlegen, werden die unterschiedlichen Leistungsindikatoren - entweder zwei Notenvariablen oder zwei Testleistungsvariablen - als Mediatoren für die Vorhersage der jeweiligen Bildungsalternativen in die Analysemodelle aufgenommen (siehe auch Abb. 1). Dadurch lässt sich quantifizieren, welche Effekte des sozialen Hintergrunds durch Leistung vermittelt werden (primäre Effekte) und welche als direkte Effekte bestehen bleiben (sekundäre Effekte). Diese Analysen werden in Anlehnung an die jüngste methodische Forschung nicht als einfache logistische Regressionen durchgeführt, sondern als sogenannte KHB-Modelle nach Karlson et al. (2012) spezifiziert. Anders als in der klassischen OLS-Regression für kontinuierliche Outcomevariablen, hängt die Skalierung der Residualvarianzen dichotomer Outcomevariablen in logistischen Regressionen von den jeweils ins Modell aufgenommenen Prädiktoren ab. Damit sind Regressionskoeffizienten zwischen logistischen Regressionsmodellen mit unterschiedlichen Prädiktoren (zusätzlichen Kovariaten) nicht miteinander vergleichbar. Durch die KHB-Methode, mit der die Residuen der weiteren Prädiktoren des Gesamtmodells auch in die reduzierten Modelle einbezogen werden, werden die Regressionsparameter vergleichbar gemacht (vgl. für eine detaillierte Darstellung der KHB-Modellgleichungen Karlson und Holm 2011). Die Effekte werden in Form von average marginal effects (AME; Karlson et al. 2012) berichtet. Im Unterschied zu odds ratios indizieren AMEs den mittleren prozentualen Zuwachs in den Übertrittswahrscheinlichkeiten, wenn sich der Prädiktor um eine Einheit verändert (unter Kontrolle der jeweiligen Kovariaten).

Die Datenanalyse fußt auf einer wesentlichen Besonderheit der KESS-Studie: den jeweiligen (Voll-)Erhebungen an den Übergangsschwellen im Bildungssystem. Um die Informationen zum Übergangsverhalten einer kompletten Kohorte eines Bundeslandes maximal auszuschöpfen, werden Querschnittsergebnisse für die einzelnen Übergangsschwellen berechnet und komparativ gegenübergestellt. Dabei werden die primären Effekte im Hinblick auf Hypothese 1 jeweils alternativ über die zu allen drei Übergängen erhobenen Noten in Mathematik und Deutsch oder über die äquivalenten, ebenfalls gemittelten, Testleistungen in Mathematik und im Leseverständnis für KESS 4 und KESS 10/11 sowie Mathematik und Englisch für KESS 12/13 operationalisiert. Die Analysen für die Studienintention werden gemäß Hypothese 4 getrennt für die gesamte Ausgangskohorte (zum Zeitpunkt KESS 10/11) und die Teilpopulation zur dritten Übergangsschwelle durchgeführt.

Die (Re-)Konstruktion der Studienintention für die Ausgangskohorte setzt dabei aufgrund der Datenlage inhaltliche Annahmen und methodische Überlegungen voraus und wird wie folgt umgesetzt: Grundsätzlich wird für alle Schüler*innen, die im Anschluss an die Sekundarstufe I nicht in eine gymnasiale Oberstufe wechselten, damit aus der KESS-Studie ausgeschieden sind und entsprechend nicht über Befragungsangaben zur Studienintention verfügen, angenommen, dass sie kein Studium anstreben (Studienintention=0). Für den übergroßen Teil dieser Gruppe dürfte dies eine plausible Annahme sein, da der Aufnahme eines Hochschulstudiums in aller Regel der Besuch einer gymnasialen Oberstufe vorangeht. Eine gewisse Unterschätzung der Studienintention dürfte jedoch auftreten, da auch andere Zugangswege zum 
Hochschulstudium bestehen, über die bereits zum Ende der Sekundarstufe I bestehende Bildungsaspirationen zum Studium (bei zunächst geringeren Schulleistungen) verfolgt werden können; genau quantifizieren lässt sich die potentielle Unterschätzung jedoch in Ermangelung entsprechender Daten nicht. Von allen zum Erhebungszeitpunkt KESS 10/11 in die Oberstufe gewechselten Schüler*innen schieden zudem während der Oberstufe bis zur KESS 12/13-Erhebung 37,7\% aus der Untersuchung aus, sodass auch für diese Schüler*innen keine Angaben zur Studienintention vorliegen. Darunter fallen vor allem Schulabgänger*innen, die ihre Schullaufbahn vorzeitig (gegebenenfalls mit dem schulischen Teil der Fachhochschulreife) beendet haben, Wiederholer*innen sowie Wechsler*innen in andere Bundesländer. Zum anderen handelt es sich hierbei um Abiturient*innen, die aus anderen Gründen (z. B. Krankheit) nicht an der abschließenden KESS-Erhebung teilgenommen haben (Teilnahmequoten in Abschn. 4.1). Von dieser Schülergruppe könnte entsprechend ein nicht unerheblicher Teil eine Studienintention aufweisen. Vor dem Hintergrund dieser Datenlage werden in den Analysen unterschiedliche Vorgehensweisen gewählt: In einer ersten Modellierung wird für die Abgänger*innen nach der Sekundarstufe I und die im Verlauf der gymnasialen Oberstufe aus der Stichprobe gefallenen Schüler*innen keine Studienintention angenommen (Studienintention=0) und sie werden mit dieser Ausprägung in den Analysen berücksichtigt. Der gewählte Ansatz auf Basis aller Schüler*innen, der fortan als inklusive Gesamtkohorte bezeichnet wird, ist als Schätzung zu verstehen und dürfte zum Teil eine Unterschätzung der Studienintention nach sich ziehen; das Vorgehen wurde daher über Robustheitsanalysen durch multiple Imputation der Studienintention für die Gruppe der während der Oberstufe aus der Stichprobe ausgeschiedenen Schüler*innen geprüft (siehe hierzu auch Diskussion, Abschn. 6). In einer zweiten Modellierung wird diese Schülergruppe aus den Analysen ausgeschlossen, sodass ausschließlich die befragten Abiturient*innen und die Schulabgänger*innen nach der 10. Jahrgangsstufe (mit der angenommenen Studienintention =0) Teil der exklusiven Gesamtkohorte sind. In allen Modellen werden zur Spezifikation der primären Effekte die Leistungsindikatoren zu KESS 10/11 verwendet, die für die gesamte Population vorliegen. In einem zusätzlichen Modell wird die Operationalisierung für einen Vergleich mit der Teilpopulation der Abiturient*innen anhand der Testleistungen in Mathematik und Englisch aus KESS 12/13 vorgenommen.

Im vorliegenden Untersuchungsdesign kam es auch zu fehlenden Werten. Der Anteil fehlender Werte lag, je nach betrachteter Variable, bei 4,2\% (Testleistungen in Mathematik und im Leseverständnis zu KESS 4) bis 43,6\% (HISEI zu KESS 12/13). ${ }^{3}$ Um systematischen Verzerrungen durch Datenausfall zu begegnen, wurde auf Basis der späteren Analysemodelle der jeweiligen Übergangsschwellen mit dem Paket mice in der Version 2.46.0 (Multiple Imputation by Chained Equations; van Buuren und Groothuis-Oudshoorn 2011) in $R$ (Version 3.4.3; R Core Team

\footnotetext{
${ }^{3}$ Die Englisch-Testleistungen zu KESS 10/11 wiesen designbedingt aufgrund eines rotierten Testheftedesigns mit 69,7\% einen höheren Anteil fehlender Werte auf: Nur einem Drittel der Schülerschaft wurde der Englisch-C-Test vorgelegt (Vieluf et al. 2011, S. 125). Gleiches gilt für Testleistungen in Sachunterricht zu KESS 4 (52,1\% fehlende Werte) und Naturwissenschaften zu KESS 12/13 (55,7\% fehlende Werte) (Bos und Pietsch 2006; Vieluf et al. 2014). Entsprechend sind die Daten als Missing Completely At Random (MCAR) anzunehmen (Lüdtke und Robitzsch 2010).
} 
2017) unter Berücksichtigung der Clusterstruktur multipel imputiert (Graham 2009; Lüdtke und Robitzsch 2010). Die hierarchische Datenstruktur wurde für KESS 4 und KESS 10/11 mit der Schulklassenzugehörigkeit und für KESS 12/13 mit der Schulzugehörigkeit als übergeordnete Ebene berücksichtigt. Zur Minimierung des Risikos systematisch fehlender Werte wurden Hilfsvariablen einbezogen, die entweder einen bedeutsamen Anteil der Varianz in den Analysevariablen aufklären konnten $(r>0,30)$ oder systematisch mit dem Datenausfall zusammenhingen (van Buuren und Groothius-Oudshoorn 2011). Es wurden pro Querschnitt 20 vollständige Datensätze generiert. Die Analyseergebnisse wurden anschließend nach Rubin (1987) gepoolt.

Die im nächsten Abschnitt berichteten Ergebnisse wurden mit robusten Standardfehlern zur Berücksichtigung der hierarchischen Datenstruktur (Schulklassen zu KESS 4 und KESS 10/11, Schulen zu KESS 12/13) mit dem Statistikprogramm Stata (Version 13.1) geschätzt. Standardfehler werden in den KHB-Modellen für die totalen und direkten Effekte, aber nicht für die indirekten Effekte geschätzt. Alle in die Modelle einbezogenen metrischen Variablen (HISEI, Schulnoten, Testleistungen) wurden zur Vergleichbarkeit der Koeffizienten zum jeweiligen Erhebungszeitpunkt $z$-standardisiert.

\section{Ergebnisse}

Die nachfolgende Ergebnisdarstellung gliedert sich entsprechend der Fragestellungen in drei Abschnitte. Dabei wird zunächst der vollständige Schülerjahrgang einbezogen, sodass die Analysen über die Übergangsschwellen hinweg vergleichbar gemacht werden. In Abschn. 5.1 wird auf Basis der Gesamtkohorte die Relation primärer und sekundärer Effekte in Abhängigkeit unterschiedlicher Leistungs- und Herkunftsindikatoren je Übergangsschwelle beschrieben. Abschn. 5.2 stellt die Veränderung des absoluten Herkunftseffektes und der primären und sekundären Anteile über die drei Übergangsschwellen hinweg dar. Unterschiede in den Befundmustern zwischen unselektierter Gesamt- und selektierter Teilpopulation an der letzten Übergangsschwelle (Studienintention) werden in Abschn. 5.3 berichtet. Dabei werden Ergebnisse auf Basis der Gesamtkohorte schwerpunktmäßig für die inklusive Vorgehensweise (siehe Abschn. 4.3) berichtet und an relevanten Stellen um Ergebnisse der exklusiven Gesamtkohorte ergänzt.

\subsection{Relation primärer und sekundärer Effekte in Abhängigkeit unterschiedlicher Leistungs- und Herkunftsindikatoren}

Die Ergebnisse zur Vorhersage der Bildungsübergänge an den drei Übergangsschwellen sowie die zugehörigen Effektzerlegungen sind in den Tab. 2, 3, 4 und 5 ausgewiesen. Modell 1 enthält jeweils die Ergebnisse unter Verwendung der Schulnoten für die Identifikation der primären Effekte, Modell 2 (und in Tab. 4 und 5 auch Modell 3) unter Verwendung der standardisierten Testleistungen. Die soziale Herkunft wird zum einen anhand des sozioökonomischen Status und zum anderen anhand des elterlichen Bildungshintergrunds abgebildet. 
Tab. 2 Dekomposition primärer und sekundärer Effekte der sozialen Herkunft am Übergang ins Gymnasium (KHB-Korrektur; average marginal effects)

\begin{tabular}{|c|c|c|c|}
\hline & $b$ & $S E$ & Anteil (in \%) \\
\hline \multicolumn{4}{|l|}{$\begin{array}{l}\text { Modell } 1 \\
\text { Mediator: Mathematik- und Deutschnote }\end{array}$} \\
\hline \multicolumn{4}{|l|}{ Herkunftseffekt: Sozialstatus } \\
\hline HISEI & 0,174 & 0,004 & \\
\hline Direkter (sekundärer) Effekt & 0,073 & 0,005 & 42,1 \\
\hline Indirekter (primärer) Effekt & 0,101 & - & 57,9 \\
\hline \multicolumn{4}{|c|}{ Herkunftseffekt: Bildungshintergrund (höchster elterlicher Schulabschluss) } \\
\hline $\begin{array}{l}\text { (Fach-)Hochschulreife (Ref.: kein Elternteil } \\
\text { mit (Fach-)Hochschulreife) }\end{array}$ & 0,339 & 0,006 & \\
\hline Direkter (sekundärer) Effekt & 0,148 & 0,008 & 43,6 \\
\hline Indirekter (primärer) Effekt & 0,191 & - & 56,4 \\
\hline \multicolumn{4}{|c|}{$\begin{array}{l}\text { Modell } 2 \\
\text { Mediatoren: Testleistungen in Mathematik und im Leseverständnis }\end{array}$} \\
\hline \multicolumn{4}{|l|}{ Herkunftseffekt: Sozialstatus } \\
\hline HISEI & 0,182 & 0,004 & \\
\hline Direkter (sekundärer) Effekt & 0,090 & 0,004 & 49,6 \\
\hline Indirekter (primärer) Effekt & 0,092 & - & 50,4 \\
\hline \multicolumn{4}{|c|}{ Herkunftseffekt: Bildungshintergrund (höchster elterlicher Schulabschluss) } \\
\hline $\begin{array}{l}\text { (Fach-)Hochschulreife (Ref.: kein Elternteil } \\
\text { mit (Fach-)Hochschulreife) }\end{array}$ & 0,350 & 0,006 & \\
\hline Direkter (sekundärer) Effekt & 0,183 & 0,007 & 52,3 \\
\hline Indirekter (primärer) Effekt & 0,167 & - & 47,7 \\
\hline
\end{tabular}

$N($ alle Modelle $)=13.873$

Datenquelle: KESS 4

An der ersten Übergangsschwelle, dem Übergang von der Grundschule in die weiterführende Schule (Tab. 2), betrug der Gesamteffekt des sozioökonomischen Status 17 (bzw. 18) Prozentpunkte ${ }^{4}$, d.h. mit einem um eine Standardabweichung höheren HISEI stieg die Wahrscheinlichkeit für den Wechsel auf das Gymnasium um 18 Prozentpunkte an. Für den elterlichen Bildungshintergrund zeigte sich, dass Grundschulkinder mit mindestens einem Elternteil mit (Fach-)Hochschulreife eine um 34 (bzw. 35) Prozentpunkte höhere Wahrscheinlichkeit für einen Gymnasialbesuch hatten als Kinder, deren Eltern einen geringeren Schulabschluss aufwiesen. Bezüglich der Relation primärer und sekundärer Effekte überwogen, mit einer Ausnahme, die indirekten (primären) Effekte: $48 \%$ (Bildungshintergrund mediiert durch Testleistungen) bis $58 \%$ (HISEI mediiert durch Schulnoten) des Gesamteffekts der sozialen Herkunft ließen sich auf primäre Effekte zurückführen. Die Differenzen in den relativen Anteilen primärer und sekundärer Effekte je nach Operationalisierung

\footnotetext{
${ }^{4}$ Die leichten Abweichungen der absoluten Effektgrößen von einem Prozentpunkt, die sich je nach verwendeten Leistungsindikatoren ergeben, sind auf das KHB-Verfahren zurückzuführen. Bei einer entsprechenden Abweichung wird der zweite Wert der Vollständigkeit halber zwar in Klammern aufgeführt, allerdings nicht gesondert interpretiert.
} 
Tab. 3 Dekomposition primärer und sekundärer Effekte der sozialen Herkunft am Übergang in die gymnasiale Oberstufe (KHB-Korrektur; average marginal effects)

\begin{tabular}{|c|c|c|c|}
\hline & $b$ & $S E$ & Anteil (in \%) \\
\hline \multicolumn{4}{|l|}{$\begin{array}{l}\text { Modell } 1 \\
\text { Mediator: Mathematik- und Deutschnote }\end{array}$} \\
\hline \multicolumn{4}{|l|}{ Herkunftseffekt: Sozialstatus } \\
\hline HISEI & 0,133 & 0,010 & \\
\hline Direkter (sekundärer) Effekt & 0,059 & 0,012 & 44,3 \\
\hline Indirekter (primärer) Effekt & 0,074 & - & 55,7 \\
\hline \multicolumn{4}{|c|}{ Herkunftseffekt: Bildungshintergrund (höchster elterlicher Schulabschluss) } \\
\hline $\begin{array}{l}\text { (Fach-)Hochschulreife (Ref.: kein Elternteil } \\
\text { mit (Fach-)Hochschulreife) }\end{array}$ & 0,276 & 0,016 & \\
\hline Direkter (sekundärer) Effekt & 0,138 & 0,020 & 50,0 \\
\hline Indirekter (primärer) Effekt & 0,138 & - & 50,0 \\
\hline \multicolumn{4}{|c|}{$\begin{array}{l}\text { Modell } 2 \\
\text { Mediatoren: Testleistungen in Mathematik und im Leseverständnis }\end{array}$} \\
\hline \multicolumn{4}{|l|}{ Herkunftseffekt: Sozialstatus } \\
\hline HISEI & 0,140 & 0,009 & \\
\hline Direkter (sekundärer) Effekt & 0,061 & 0,008 & 43,5 \\
\hline Indirekter (primärer) Effekt & 0,079 & - & 56,6 \\
\hline \multicolumn{4}{|c|}{ Herkunftseffekt: Bildungshintergrund (höchster elterlicher Schulabschluss) } \\
\hline $\begin{array}{l}\text { (Fach-)Hochschulreife (Ref.: kein Elternteil } \\
\text { mit (Fach-)Hochschulreife) }\end{array}$ & 0,285 & 0,015 & \\
\hline Direkter (sekundärer) Effekt & 0,152 & 0,013 & 53,4 \\
\hline Indirekter (primärer) Effekt & 0,133 & - & 46,6 \\
\hline
\end{tabular}

$N($ alle Modelle $)=12.551$

Datenquelle: KESS 10/11

der leistungsbezogenen Effekte fielen moderat aus, deuten jedoch auf eine Unterschätzung sekundärer Herkunftseffekte hin, wenn primäre Effekte über Schulnoten operationalisiert werden (Hypothese 1): Sowohl für den HISEI als auch für den Bildungshintergrund fiel der Anteil sekundärer Effekte bei Verwendung der Schulnoten gegenüber den Testleistungen 8 Prozentpunkte geringer aus.

Tab. 3 enthält die Ergebnisse für den Übergang in die gymnasiale Oberstufe. An dieser Übergangsschwelle lag der absolute Herkunftseffekt für den sozioökonomischen Status bei 13 (bzw. 14) Prozentpunkten, für den Bildungshintergrund bei 28 (bzw. 29) Prozentpunkten. Bezüglich der Relation primärer und sekundärer Herkunftseffekte zeigte sich, dass primäre Effekte für den sozioökonomischen Status größer ausfielen als für den Bildungshintergrund. Dies galt sowohl für Schulnoten (56 vs. $50 \%$ ) als auch Testleistungen (57 vs. $47 \%$ ) als Mediator. Zur Frage, ob die Operationalisierung der primären Effekte durch Schulnoten oder Testleistungen unterschiedliche Anteile primärer und sekundärer Effekte bewirkt, fielen die Befunde uneinheitlich aus. Während für den Bildungshintergrund als Herkunftsindikator kleinere Anteile primärer Effekte für die Testleistungen (47 vs. 50\% für die Schulnoten) resultierten, fielen beim HISEI die primären Effekte für die Testleistungen geringfügig größer aus als für die Schulnoten (57 vs. 56\%). 
Tab. 4 Dekomposition primärer und sekundärer Effekte der sozialen Herkunft am Übergang nach der Sekundarstufe II (Studienintention), Gesamtkohorte inklusiv (KHB-Korrektur; average marginal effects)

\begin{tabular}{|c|c|c|c|}
\hline & $b$ & $S E$ & Anteil (in \%) \\
\hline \multicolumn{4}{|l|}{$\begin{array}{l}\text { Modell } 1 \\
\text { Mediatoren: Mathematik- und Deutschnote }\end{array}$} \\
\hline \multicolumn{4}{|l|}{ Herkunftseffekt: Sozialstatus } \\
\hline HISEI & 0,102 & 0,006 & \\
\hline Direkter (sekundärer) Effekt & 0,052 & 0,008 & 51,2 \\
\hline Indirekter (primärer) Effekt & 0,050 & - & 48,8 \\
\hline \multicolumn{4}{|c|}{ Herkunftseffekt: Bildungshintergrund (höchster elterlicher Schulabschluss) } \\
\hline $\begin{array}{l}\text { (Fach-)Hochschulreife (Ref.: kein Elternteil } \\
\text { mit (Fach-)Hochschulreife) }\end{array}$ & 0,230 & 0,011 & \\
\hline Direkter (sekundärer) Effekt & 0,138 & 0,012 & 60,0 \\
\hline Indirekter (primärer) Effekt & 0,092 & - & 40,0 \\
\hline \multicolumn{4}{|c|}{$\begin{array}{l}\text { Modell } 2 \\
\text { Mediatoren: Testleistungen in Mathematik und im Leseverständnis }\end{array}$} \\
\hline \multicolumn{4}{|l|}{ Herkunftseffekt: Sozialstatus } \\
\hline HISEI & 0,099 & 0,006 & \\
\hline Direkter (sekundärer) Effekt & 0,048 & 0,005 & 48,5 \\
\hline Indirekter (primärer) Effekt & 0,051 & - & 51,5 \\
\hline \multicolumn{4}{|c|}{ Herkunftseffekt: Bildungshintergrund (höchster elterlicher Schulabschluss) } \\
\hline $\begin{array}{l}\text { (Fach-)Hochschulreife (Ref.: kein Elternteil } \\
\text { mit (Fach-)Hochschulreife) }\end{array}$ & 0,224 & 0,010 & \\
\hline Direkter (sekundärer) Effekt & 0,138 & 0,010 & 61,8 \\
\hline Indirekter (primärer) Effekt & 0,085 & - & 38,2 \\
\hline \multicolumn{4}{|c|}{$\begin{array}{l}\text { Modell } 3 \\
\text { Mediatoren: Testleistungen in Mathematik und in Englisch }\end{array}$} \\
\hline \multicolumn{4}{|l|}{ Herkunftseffekt: Sozialstatus } \\
\hline HISEI & 0,098 & 0,006 & \\
\hline Direkter (sekundärer) Effekt & 0,040 & 0,006 & 41,1 \\
\hline Indirekter (primärer) Effekt & 0,058 & - & 58,9 \\
\hline \multicolumn{4}{|c|}{ Herkunftseffekt: Bildungshintergrund (höchster elterlicher Schulabschluss) } \\
\hline $\begin{array}{l}\text { (Fach-)Hochschulreife (Ref.: kein Elternteil } \\
\text { mit (Fach-)Hochschulreife) }\end{array}$ & 0,223 & 0,010 & \\
\hline Direkter (sekundärer) Effekt & 0,124 & 0,011 & 55,7 \\
\hline Indirekter (primärer) Effekt & 0,099 & - & 44,3 \\
\hline
\end{tabular}

$N($ alle Modelle $)=12.551$

Datenquelle: KESS 10/11 und KESS 12/13

Herkunftseffekte auf die Studienintention sind in Tab. 4 auf Basis der inklusiven Gesamtkohorte und in Tab. 5 auf Basis der exklusiven Gesamtkohorte aufgeführt (vgl. für die Gegenüberstellung mit der selektierten Teilstichprobe Abschn. 5.3). Der absolute Effekt des sozioökonomischen Status für die inklusive Gesamtkohorte betrug 10 Prozentpunkte, der Effekt des familiären Bildungshintergrunds 22 (bzw. 23) Prozentpunkte. Bezüglich der Relation primärer und sekundärer Effekte auf die Studienintention überwog der sekundäre Effekt mit Anteilen bis zu $62 \%$ ausschließlich für den familiären Bildungshintergrund. Effekte des sozioökonomischen Status 
Tab. 5 Dekomposition primärer und sekundärer Effekte der sozialen Herkunft am Übergang nach der Sekundarstufe II (Studienintention), Gesamtkohorte exklusiv (KHB-Korrektur; average marginal effects)

\begin{tabular}{|c|c|c|c|}
\hline & $b$ & $S E$ & Anteil (in \%) \\
\hline \multicolumn{4}{|l|}{$\begin{array}{l}\text { Modell } 1 \\
\text { Mediatoren: Mathematik- und Deutschnote }\end{array}$} \\
\hline \multicolumn{4}{|l|}{ Herkunftseffekt: Sozialstatus } \\
\hline HISEI & 0,148 & 0,008 & \\
\hline Direkter (sekundärer) Effekt & 0,073 & 0,010 & 49,6 \\
\hline Indirekter (primärer) Effekt & 0,074 & - & 50,4 \\
\hline \multicolumn{4}{|c|}{ Herkunftseffekt: Bildungshintergrund (höchster elterlicher Schulabschluss) } \\
\hline $\begin{array}{l}\text { (Fach-)Hochschulreife (Ref.: kein Elternteil } \\
\text { mit (Fach-)Hochschulreife) }\end{array}$ & 0,317 & 0,012 & \\
\hline Direkter (sekundärer) Effekt & 0,180 & 0,017 & 56,6 \\
\hline Indirekter (primärer) Effekt & 0,138 & - & 43,4 \\
\hline \multicolumn{4}{|c|}{$\begin{array}{l}\text { Modell } 2 \\
\text { Mediatoren: Testleistungen in Mathematik und im Leseverständnis }\end{array}$} \\
\hline \multicolumn{4}{|l|}{ Herkunftseffekt: Sozialstatus } \\
\hline HISEI & 0,146 & 0,007 & \\
\hline Direkter (sekundärer) Effekt & 0,068 & 0,006 & 46,2 \\
\hline Indirekter (primärer) Effekt & 0,079 & - & 53,8 \\
\hline \multicolumn{4}{|c|}{ Herkunftseffekt: Bildungshintergrund (höchster elterlicher Schulabschluss) } \\
\hline $\begin{array}{l}\text { (Fach-)Hochschulreife (Ref.: kein Elternteil } \\
\text { mit (Fach-)Hochschulreife) }\end{array}$ & 0,312 & 0,012 & \\
\hline Direkter (sekundärer) Effekt & 0,178 & 0,012 & 57,1 \\
\hline Indirekter (primärer) Effekt & 0,134 & - & 42,9 \\
\hline \multicolumn{4}{|c|}{$\begin{array}{l}\text { Modell } 3 \\
\text { Mediatoren: Testleistungen in Mathematik und in Englisch }\end{array}$} \\
\hline \multicolumn{4}{|l|}{ Herkunftseffekt: Sozialstatus } \\
\hline HISEI & 0,145 & 0,006 & \\
\hline Direkter (sekundärer) Effekt & 0,054 & 0,006 & 37,2 \\
\hline Indirekter (primärer) Effekt & 0,091 & - & 62,8 \\
\hline \multicolumn{4}{|c|}{ Herkunftseffekt: Bildungshintergrund (höchster elterlicher Schulabschluss) } \\
\hline $\begin{array}{l}\text { (Fach-)Hochschulreife (Ref.: kein Elternteil } \\
\text { mit (Fach-)Hochschulreife) }\end{array}$ & 0,310 & 0,010 & \\
\hline Direkter (sekundärer) Effekt & 0,154 & 0,011 & 49,7 \\
\hline Indirekter (primärer) Effekt & 0,156 & - & 50,3 \\
\hline
\end{tabular}

$N($ alle Modelle $)=12.551$

Datenquelle: KESS 10/11 und KESS 12/13

wurden hingegen in etwa zu gleichen Anteilen von primären und sekundären Effekten erklärt. Unterschiede in den relativen Größen primärer und sekundärer Effekte je nach Operationalisierung des primären Effekts zeigten sich auch am Übergang zur Hochschule. Primäre Effekte des Bildungshintergrunds fielen für die Testleistungen im Vergleich zu den Schulnoten etwas kleiner aus (38 vs. 40\%), primäre Effekte des sozioökonomischen Status fielen für die Testleistungen vergleichsweise größer aus (52 vs. $49 \%$ ). Ein Vergleich mit der exklusiven Gesamtkohorte zeigt, dass die primären Effekte des sozioökonomischen Status bei diesem Vorgehen um rund 2 
bis 3 Prozentpunkte größer ausfielen, für den Bildungshintergrund um 3 bis 5 Prozentpunkte. Der sekundäre Effekt für den Bildungshintergrund nimmt unabhängig von der Operationalisierung primärer Effekte einen relativen Anteil von $57 \%$ an, während sich in der inklusiven Gesamtkohorte eine kleine Differenz von 2 Prozentpunkten ergab (60\% Schulnoten vs. $62 \%$ Testleistungen). Der sekundäre Effekt für den sozioökonomischen Status beläuft sich in der exklusiven Gesamtkohorte auf $50 \%$ für die Schulnoten und auf $46 \%$ für die Testleistungen, während sich in der inklusiven Gesamtkohorte eine etwas geringere Differenz von 2 Prozentpunkten fand (51\% Schulnoten vs. $49 \%$ Testleistungen). Es deuten sich also je nach zugrunde gelegter Gesamtkohorte (inklusiv/exklusiv) leichte Abweichungen an, grundlegende Veränderungen in den Ergebnismustern sind jedoch nicht auszumachen.

\subsection{Veränderung des absoluten Herkunftseffektes und der primären und sekundären Anteile über die drei Übergangsschwellen hinweg}

Ein Vergleich der in den KHB-Modellen geschätzten Effektgrößen an den drei Übergangsschwellen, die in Abb. 2 zusammenfassend veranschaulicht sind, zeigt über den Verlauf der Sekundarstufe eine kontinuierliche Abnahme des absoluten Herkunftseffekts auf den jeweiligen Bildungsübergang (Hypothese 2). Während der Herkunftseffekt beim Wechsel in das Gymnasium wie beschrieben bis zu 18 Prozentpunkte (HISEI) bzw. 35 Prozentpunkte (Bildungshintergrund) betrug, hat er sich an den weiteren Übergangsschwellen auf bis zu 22 bzw. 10 Prozentpunkte in der Erklärung der Studienintention für die inklusive Gesamtkohorte verringert. Für die exklusive Gesamtkohorte zeigte sich dieser Befund hingegen nicht, da der Herkunftseffekt für den sozioökonomischen Status 15 Prozentpunkte und für den Bildungshintergrund

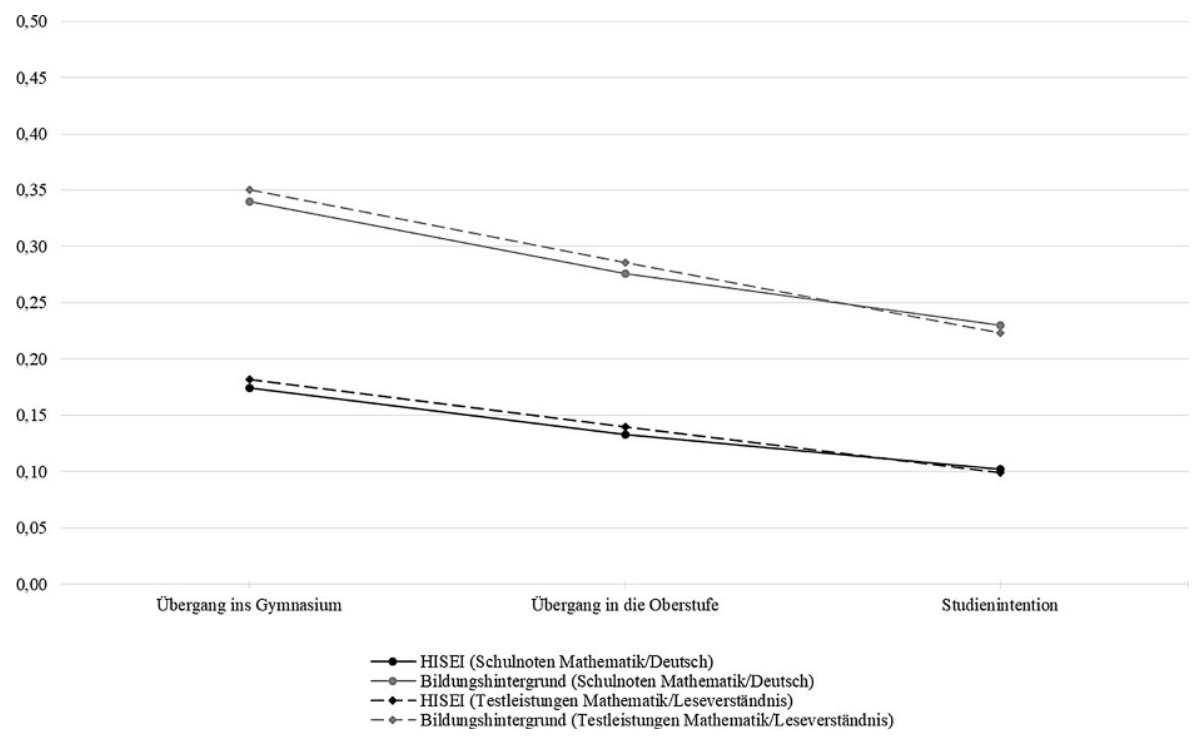

Abb. 2 Veränderung des absoluten Herkunftseffekts (average marginal effects) über den Verlauf der Sekundarstufe (Gesamtkohorte inklusiv) 


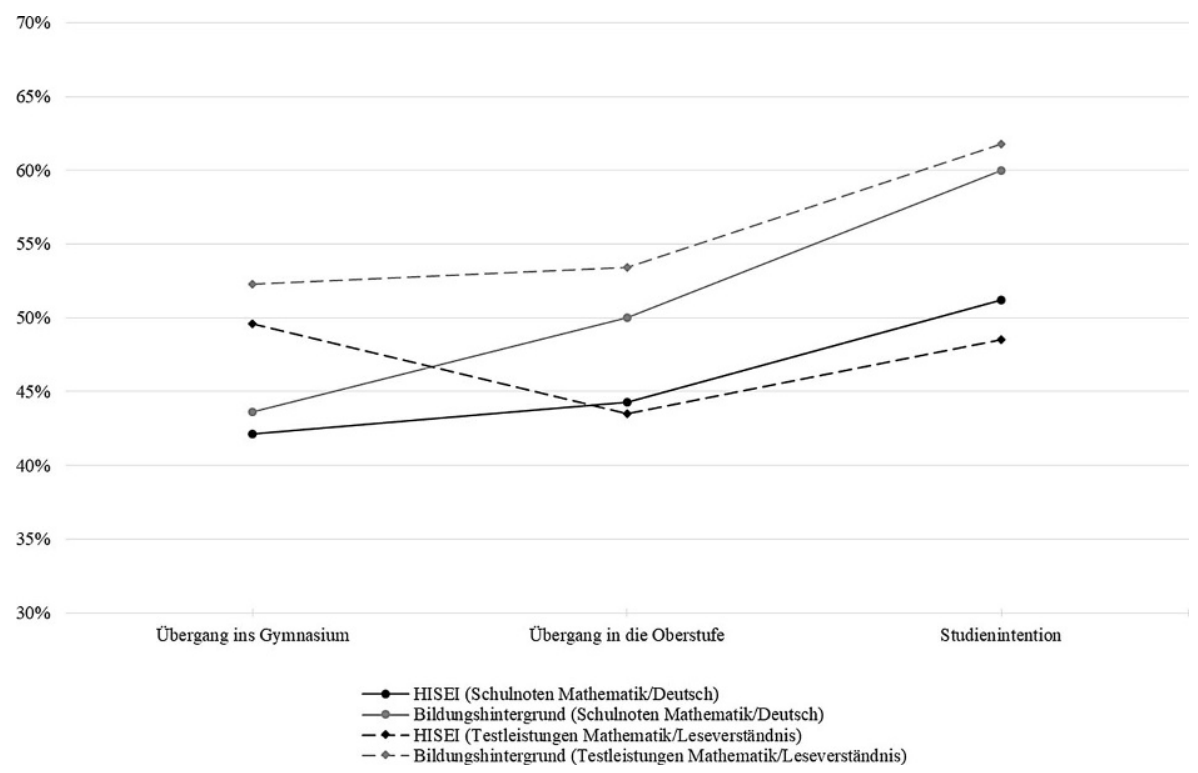

Abb. 3 Anteil sekundärer Effekte am Herkunftseffekt über den Verlauf der Sekundarstufe (Gesamtkohorte inklusiv)

31 (bzw. 32) Prozentpunkte betrug und sich damit sogar zwischen den ermittelten Herkunftseffekten am Übergang auf das Gymnasium und in die gymnasiale Oberstufe einordnete.

Das relative (prozentuale) Gewicht des sekundären Effekts wird in Abb. 3 über die fokussierten Zeitpunkte für die Gesamtkohorte komparativ dargestellt. Die schwellenvergleichende Betrachtung des Ausmaßes sekundärer Effekte ergab für den HISEI je nach verwendetem Leistungsmaß eine Spanne von 42 bis $51 \%$, wobei die niedrigsten Werte (44\%) für den Übergang in die Oberstufe resultierten, die tendenziell höchsten Werte für die Studienintention (49 bzw. 51\%). Die Werte für den Übergang ans Gymnasium bewegten sich dazwischen (50 bzw. 42\%). Insgesamt sind damit für den HISEI weder deutliche Unterschiede zwischen den Übergangsschwellen noch ein Entwicklungsverlauf im Sinne sukzessiv steigender sekundärer Effekte feststellbar. Ein leicht abgewandeltes Muster fand sich hingegen, wenn anstelle des HISEI der elterliche Bildungshintergrund herangezogen wird. Hier zeigten sich mit Anteilen von 62 bzw. $60 \%$ substanziell höhere Anteile sekundärer Effekte auf die Studienintention als für den Übergang in das Gymnasium (52 bzw. 44\%) oder die Oberstufe (53 bzw. 50\%). Für den elterlichen Bildungshintergrund deutet sich somit eine Zunahme des sekundären Herkunftseffektes an, wenngleich herauszustellen ist, dass es sich nicht um eine sukzessive ansteigende Entwicklung handelt, sondern in erster Linie um einen Anstieg zwischen den beiden späteren Übergangsschwellen. Zudem lagen die sekundären Effekte immer noch unter den bisherigen Befunden bei Heranziehung selektierter Teilpopulationen (vgl. dazu den nachfolgenden Abschn. 5.3). Über den gesamten Verlauf der Sekundarstufe wurde mit den vorliegenden Befunden auf Basis der vollständigen Schülerkohorte für das Bundes- 
land Hamburg folglich keine schrittweise rückläufige Bedeutung primärer Effekte bzw. schrittweise steigende relative Bedeutung sekundärer Herkunftseffekte nachgewiesen (Hypothese 3). Erst zur Frage, ob ein Hochschulstudium angestrebt wird, fiel die Relevanz sekundärer Effekte etwas höher aus und ist auf das Effektmuster für den familiären Bildungshintergrund beschränkt. Dieses schwellenvergleichende Befundmuster lässt sich auch dann aufrechterhalten, wenn die exklusive Gesamtkohorte zugrunde gelegt wird, wenngleich der relative Bedeutungszuwachs sekundärer Effekte zur Studienintention etwas moderater ausfiel (vgl. Abschn. 5.1).

\subsection{Unterschiede in den Befundmustern zwischen Gesamt- und selektierter Teilpopulation}

Mit Blick auf mögliche Unterschiede in den Befundmustern zur Studienintention zwischen der vollständigen Ausgangskohorte (Hypothese 4, Tab. 4, für einen Vergleich unter Operationalisierung primärer Effekte anhand der Testleistungen in Mathematik und Englisch siehe Modell 3) und der selektierten Teilpopulation der Abiturient*innen (Tab. 6) konnte festgestellt werden, dass die absoluten Herkunftseffekte innerhalb der selektiven Schülergruppe zum Ende der 12. bzw. 13. Jahrgangsstufe für beide Formen der sozialen Herkunft im Vergleich zur gesamten Ausgangskohorte (inklusiv) geringer ausfielen (Sozialstatus: 5 vs. 10 Prozentpunkte; Bildungshintergrund: 14 vs. 22 [bzw. 23] Prozentpunkte). Die relativen Anteile primärer und sekundärer Effekte an den Herkunftseffekten sind in Abb. 4 für Gesamtund Teilpopulation gegenübergestellt. Die relativen Anteile sekundärer Effekte der Teilpopulation reichten für den HISEI von $68 \%$ (Mediator: Schulnoten) bis $75 \%$

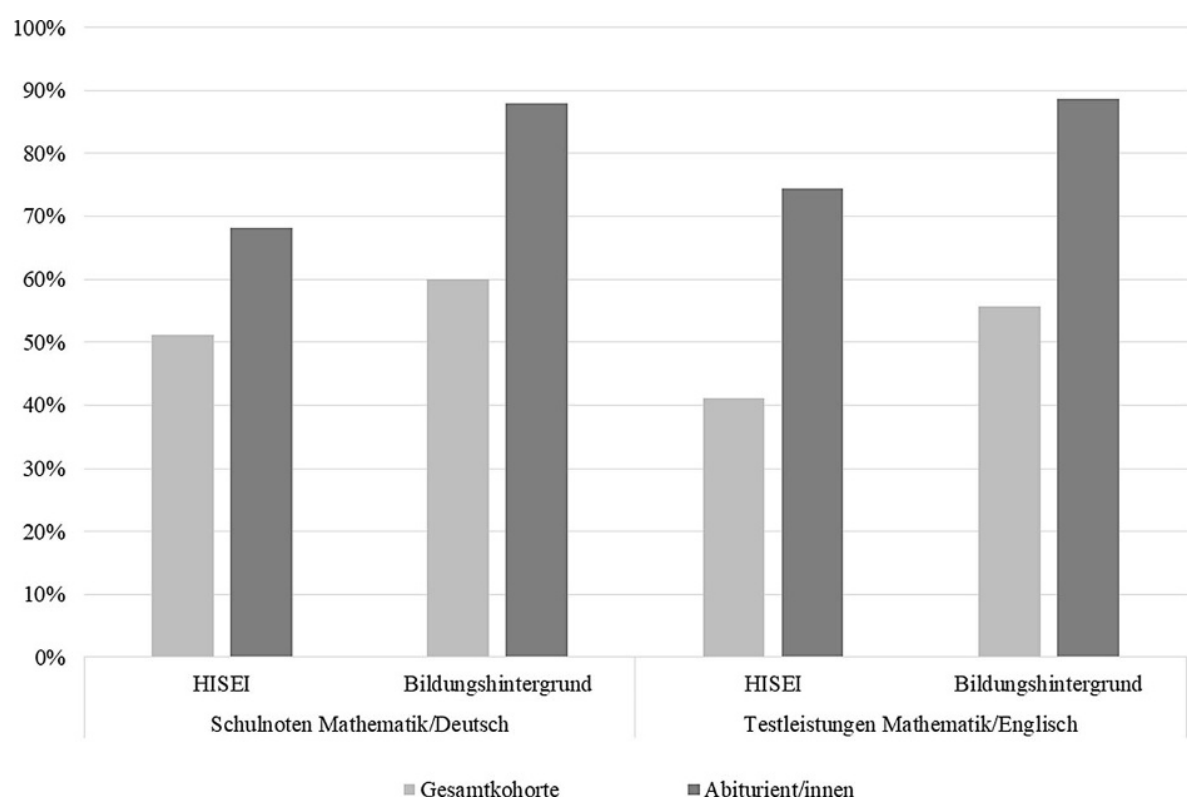

Abb. 4 Anteil sekundärer Effekte am Herkunftseffekt auf die Studienintention, Gesamtkohorte (inklusiv) und Abiturient*innen im Vergleich 
Tab. 6 Dekomposition primärer und sekundärer Effekte der sozialen Herkunft am Übergang nach der Sekundarstufe II (Studienintention), Teilpopulation Abiturient*innen (KHB-Korrektur; average marginal effects)

\begin{tabular}{|c|c|c|c|}
\hline & $b$ & $S E$ & Anteil (in \%) \\
\hline \multicolumn{4}{|l|}{$\begin{array}{l}\text { Modell } 1 \\
\text { Mediator: Mathematik- und Deutschnote }\end{array}$} \\
\hline \multicolumn{4}{|l|}{ Herkunftseffekt: Sozialstatus } \\
\hline HISEI & 0,045 & 0,008 & \\
\hline Direkter (sekundärer) Effekt & 0,030 & 0,008 & 68,1 \\
\hline Indirekter (primärer) Effekt & 0,014 & - & 31,9 \\
\hline \multicolumn{4}{|c|}{ Herkunftseffekt: Bildungshintergrund (höchster elterlicher Schulabschluss) } \\
\hline $\begin{array}{l}\text { (Fach-)Hochschulreife (Ref.: kein Elternteil } \\
\text { mit (Fach-)Hochschulreife) }\end{array}$ & 0,143 & 0,015 & \\
\hline Direkter (sekundärer) Effekt & 0,126 & 0,015 & 87,9 \\
\hline Indirekter (primärer) Effekt & 0,017 & - & 12,1 \\
\hline \multicolumn{4}{|c|}{$\begin{array}{l}\text { Modell } 2 \\
\text { Mediatoren: Testleistungen in Mathematik und Englisch }\end{array}$} \\
\hline \multicolumn{4}{|l|}{ Herkunftseffekt: Sozialstatus } \\
\hline HISEI & 0,045 & 0,009 & \\
\hline Direkter (sekundärer) Effekt & 0,033 & 0,008 & 74,5 \\
\hline Indirekter (primärer) Effekt & 0,011 & - & 25,5 \\
\hline \multicolumn{4}{|c|}{ Herkunftseffekt: Bildungshintergrund (höchster elterlicher Schulabschluss) } \\
\hline $\begin{array}{l}\text { (Fach-)Hochschulreife (Ref.: kein Elternteil } \\
\text { mit (Fach-)Hochschulreife) }\end{array}$ & 0,144 & 0,016 & \\
\hline Direkter (sekundärer) Effekt & 0,127 & 0,015 & 88,6 \\
\hline Indirekter (primärer) Effekt & 0,016 & - & 11,4 \\
\hline
\end{tabular}

$N($ alle Modelle $)=6632$

Datenquelle: KESS 12/13

(Mediator: Testleistungen in Mathematik und Englisch) und 88 bis $89 \%$ für Effekte des Bildungshintergrunds. Die Anteile sekundärer Herkunftseffekte fielen somit unter Zugrundelegung der Teilstichprobe der Abiturient*innen deutlich höher aus als für die inklusive Gesamtkohorte und insbesondere im Vergleich zur exklusiven Gesamtkohorte (vgl. Abschn. 5.1).

\section{Zusammenfassung und Diskussion}

Gegenstand der vorliegenden Studie war die Untersuchung sozialer Herkunftseffekte an drei Übergangsschwellen im Verlauf der Sekundarstufe: dem Übergang ins Gymnasium und die Oberstufe sowie die Intention zur Aufnahme eines Hochschulstudiums. Neben den absoluten Herkunftseffekten und ihrer Entwicklung über die aufeinanderfolgenden Bildungsübergänge stand dabei vor allem die Frage nach der relativen Bedeutung primärer und sekundärer Herkunftseffekte im Zentrum. Auf der Datengrundlage der Hamburger KESS-Studie wurden sowohl unterschiedliche soziale Herkunftsmaße (HISEI und Bildungshintergrund) als auch Leistungsindikatoren (Tests und Schulnoten) einbezogen und hinsichtlich ihrer Auswirkungen auf die An- 
teilsrelationen untersucht. Zu allen drei Bildungsübergängen konnten die Analysen zudem auf Basis der jeweils (fast) vollständigen Schülerschaft durchgeführt werden, was die Befunde über den Verlauf der Sekundarstufe hinweg vergleichbar macht. Eine weitere Besonderheit der Studie bestand darin, Effekte der sozialen Herkunft an der dritten Übergangsschwelle (Studienintention) sowohl - wie bislang üblich - für die Teilpopulation der Abiturient*innen als auch auf Grundlage der Gesamt- bzw. Ausgangskohorte zu quantifizieren und gegenüberzustellen. Im Folgenden sollen die Befunde zusammengefasst und diskutiert werden.

Mit der vorliegenden Untersuchung war es zunächst unter einer generellen Perspektive möglich, die These abnehmender sozialer Herkunftseffekte über den Bildungsverlauf an drei Bildungsübergängen im Verlauf der Sekundarstufe zu prüfen. Theoretisch argumentiert wird zum einen mit einer zunehmenden Selektion der Schülerschaft an den aufeinanderfolgenden Übergangsschwellen, zum anderen mit einem sinkenden Einfluss der Herkunftsfamilie im Lebensverlauf der Schüler*innen (vgl. Blossfeld und Shavit 1993; Mare 1980). Wie die Ergebnisse der vorliegenden Studie zeigten, hat sich der totale soziale Herkunftseffekt auf das Übergangsverhalten vom Übergang von der Grund- in die Sekundarschule, über den Eintritt in die gymnasiale Oberstufe bis zur Studienintention verringert, wenn die selektive Stichprobe der Abiturient*innen zugrunde gelegt wird. Diese Entwicklung gilt für beide verwendeten Maße zur Operationalisierung der sozialen Herkunft: den sozioökonomischen Status der Familie (HISEI), gemessen über eine standardisierte kontinuierliche Variable, sowie die kulturellen Ressourcen der Eltern, die über den höchsten Schulabschluss der Eltern mit einer dichotomen Variable (Vorliegen einer (Fach-)Hochschulreife) in gröberer Form abgebildet wurden. Bisherige Befunde im deutschen Kontext (vgl. etwa Becker 2009; Neugebauer et al. 2013) werden damit bestätigt. Eine Erweiterung des Forschungsstandes ergibt sich aus der vergleichenden Betrachtung der Befunde für die Studienintention auf Basis der selektierten Teilpopulation und der Gesamtpopulation, womit gleichzeitig Selektions- und Lebensverlaufseffekte genauer in den Blick genommen werden konnten. Dabei zeigte sich ein differenziertes Muster in Abhängigkeit des gewählten Vorgehens zur Abbildung der Gesamtkohorte. Wurde für alle Personen ohne Angabe einer Studienintention der Wert Null angenommen (inklusive Gesamtkohorte), zeigte sich auch hier das Muster rückläufiger absoluter Herkunftseffekte über die Übergangsschwellen. Wurden stattdessen Personen ausgeschlossen, die im Laufe der gymnasialen Oberstufe aus der Stichprobe ausgeschieden sind (exklusive Gesamtkohorte), verringerten sich die absoluten Herkunftseffekte hingegen nicht. Die Annahme abnehmender Herkunftseffekte über den Verlauf der Sekundarstufe lässt sich somit uneingeschränkt nur für die selektive Analysegruppe der Abiturient*innen bestätigen, während sich mit Blick auf die Gesamtkohorte kein klares Bild ergibt, da hier zum Teil auch gleichbleibende Herkunftseffekte indiziert werden.

Neben der generellen Ungleichheitsperspektive ging die vorliegende Untersuchung der sowohl für die Bildungsforschung als auch für die Bildungspraxis wichtigen Frage nach, welche relative Bedeutung primäre und sekundäre Effekte der sozialen Herkunft (Boudon 1974) im Speziellen über den Verlauf der Sekundarstufe einnehmen: Wie stark und an welcher Übergangsschwelle verändert sich die Relation beider Effekte? Während die Effektdekomposition nahezu unabhängig von den 
Operationalisierungen der sozialen Herkunft und der Schulleistungen ein leichtes Übergewicht primärer Effekte beim Übergang in das Gymnasium ergab, ist das Bild an der nächsten Übergangsschwelle uneinheitlich. Gemessen an den standardisierten Testleistungen stieg die relative Bedeutung primärer Effekte beim Übergang in die gymnasiale Oberstufe sogar leicht (von 50 auf 57\%, Herkunftseffekt über HISEI); alle übrigen Veränderungen sind marginal. Somit verweist die für diese Übergangsschwelle erstmals umgesetzte Quantifizierung primärer und sekundärer Effekte erstens darauf, dass leistungsbezogene Herkunftseffekte auch für den Eintritt in die gymnasiale Oberstufe relevant sind. Zweitens wird durch die Analyse dieser Übergangsschwelle sichtbar, dass sekundäre Effekte erst an der unmittelbaren Schwelle $\mathrm{zu}$ tertiärer Bildung, relativ gesehen, eine bedeutendere Rolle zu spielen scheinen. Für die Gesamtkohorte der Studie geht der Effekt des familiären Bildungshintergrunds auf die Studienintention zu rund $57 \%$ (exklusive Gesamtkohorte) bis $60 \%$ (inklusive Gesamtkohorte) auf sekundäre Effekte zurück. Für die selektierte Teilpopulation der Abiturient*innen beruht der Effekt der elterlichen Bildung auf die Intention, ein Studium zu beginnen, hingegen zu fast $90 \%$ auf nicht-leistungsbezogenen Aspekten. Zur Einordnung dieser und bisheriger Befunde zur Rolle primärer und sekundärer Herkunftseffekte an der Schwelle zum Studium bedeutet dies, dass herkunftsbedingte Leistungsunterschiede durchaus relevant für die Studienintention sind, wenn die Perspektive auf die Gesamtkohorte erweitert wird. Allerdings hat sich auf dieser Grundlage zugleich die übergeordnete Rolle des sekundären Effekts am Übergang in tertiäre Bildung bestätigt.

Die im Forschungsstand identifizierten Implikationen zu methodischen Fragen der Quantifizierung primärer und sekundärer Effekte und diesbezüglichen Forschungsdesiderata konnten im Rahmen der Studie aufgegriffen werden. Durch den Einsatz verschiedener Operationalisierungen ließ sich zunächst herausstellen, welche Implikationen für die empirische Analyse der Bildungsungleichheiten von den Messinstrumenten ausgehen. So konnte etwa eine mögliche Unterschätzung sekundärer Effekte bei Verwendung der Schulnoten zur Messung des indirekten (primären) Effekts (vgl. Maaz und Nagy 2009) nur mit leichter Tendenz am Übergang von der Grund- in die Sekundarstufe aufgezeigt werden. Wenn die Schülerleistung anhand von objektiveren Testleistungen operationalisiert wird, werden die mittlerweile unter dem Begriff tertiärer Effekte (vgl. Esser 2016) diskutierten Einflüsse einer schichtspezifischen Schülerbeurteilung durch die Lehrkräfte möglicherweise besser berücksichtigt, wenngleich sie in den hier dargelegten Modellen Teil der sekundären Effekte sind. Zur Frage, welche Übergangsschwellen zur Quantifizierung von Herkunftseffekten verglichen werden sollten (und basierend auf welcher Schülerpopulation), um zu einer möglichst umfassenden Darstellung (der Veränderung) von Bildungsungleichheiten zu gelangen, kann die Studie neue Diskussionspunkte liefern. Erstens kann festgehalten werden, dass unter Einbezug des Übergangs in die Oberstufe ein tatsächlicher Verlauf von Herkunftseffekten dargestellt werden kann. Deutlich wurde nach diesen Ergebnissen vor allem, dass sich die relativen Größen primärer und sekundärer Effekte an den ersten beiden Übergangsschwellen ähneln. Werden die Analysen auch auf die Gesamtkohorte bezogen, eröffnet sich zweitens die Möglichkeit, Herkunftseffekte über Selektionseffekte an späteren Übergangsschwellen hinausgehend vollständiger abzubilden. Dieser Ansatz, d.h. die bloße 
Lebensverlaufsperspektive ohne Einschränkungen aufgrund der zunehmend selektiven Stichprobenzusammensetzung an den späteren Übergangsschwellen, beugt nach den vorgestellten Befunden einer Unterschätzung primärer Effekte vor.

Zur weiteren Einordnung der Befunde wurden zusätzliche Robustheitschecks durchgeführt, um den Einfluss der gewählten (über die Übergangsschwellen möglichst uniformen) Leistungsindikatoren herauszustellen. Zum Zwecke der Vergleichbarkeit über die verschiedenen Übergänge hinweg, wurden in unseren Analysen jeweils gleichartige Leistungsindikatoren (Testleistungen und Noten in Deutsch und Mathematik) herangezogen. Gleichwohl ist denkbar, dass die Befunde für die einzelnen Übergänge in Teilen modifiziert werden, wenn weitere - jeweils übergangsspezifisch verfügbare - Leistungsindikatoren einbezogen werden. Die Tab. A1 bis A3a/b/c im Anhang weisen (analog zu den Tab. 2, 3, 4, 5 und 6) Ergebnisse aus, die auf maximalen Informationen über die Schulleistungen für die einzelnen Übergänge beruhen. Ein Vergleich der relativen Größen primärer und sekundärer Effekte zeigt: Wurden mehr Leistungsindikatoren als Mediatoren zur Ermittlung des primären Effekts in die KHB-Modelle integriert, fiel dieser in der Regel um einige Prozentpunkte gröBer aus (0 bis 5 Prozentpunkte) - das grundlegende Befundmuster ändert sich in diesen Fällen jedoch nicht. In einzelnen Fällen sind hingegen größere Verschiebungen feststellbar, die insbesondere aus dem Einbezug der Testleistungen in Englisch resultierten. So zeigte sich beim Übergang in die gymnasiale Oberstufe ein um 11 Prozentpunkte (HISEI) bzw. 13 Prozentpunkte (Bildungshintergrund) größerer primärer Effekt, wenn zusätzlich die Testleistungen in Englisch einbezogen wurden. Ähnlich große Differenzen (zwischen 8 und 11 Prozentpunkten) ergaben sich hinsichtlich der Studienintention auf Basis der Gesamtkohorte (inklusiv/exklusiv), sodass sich primäre und sekundäre Effekte in etwa die Waage hielten: Mit differentiellen Fremdsprachenkenntnissen beim Übergang in sowie im Anschluss an die gymnasiale Oberstufe sind also bedeutsame zusätzliche primäre Effekte verbunden. Für die Teilpopulation der Abiturient*innen fiel der primäre Effekt auf die Studienintention deutlich größer aus (HISEI: 10 Prozentpunkte; Bildungshintergrund: 8 Prozentpunkte), wenn ein breites Spektrum an Noten berücksichtigt wurde. Dennoch überwog weiterhin der sekundäre Effekt der differentiellen Bildungsentscheidungen, für den Bildungshintergrund mit rund $80 \%$ sogar deutlich. Als Fazit dieser Robustheitsanalysen lässt sich resümieren, dass der Einbezug weiterer Leistungsmerkmale teilweise zu höheren Anteilen für die primären Effekte führen kann, jedoch nur vereinzelt Unterschiede in den grundlegenden Befundmustern feststellbar sind.

\subsection{Grenzen und Ausblick}

Wie oben dargelegt, ist es eine Stärke der vorliegenden Studie, dass zu jedem Erhebungszeitpunkt und somit jeder Übergangsschwelle die vollständige (bzw. zu KESS 12/13 nahezu vollständige) Schülerkohorte zugrunde gelegt werden konnte, um Querschnittsvergleiche aller Schüler*innen vornehmen zu können. Dies führt jedoch zugleich zu einigen Limitationen dieser Studie. Über den gesamten Untersuchungszeitraum standen zwar die Schulnoten in vergleichbaren Fächern zur Verfügung, nicht aber die äquivalenten Testleistungen zum Zeitpunkt KESS 12/13. Hier musste von den Deutsch- auf die Englischleistungen als Maß für sprachliche Kompe- 
tenzen ausgewichen werden. Für die Analyse der Studienintention (Gesamtkohorte) am Ende der gymnasialen Oberstufe gilt ferner, dass die schulischen Leistungen zum Zeitpunkt KESS 10/11 als Mediatoren indirekter Effekte in die KHB-Modelle einfließen mussten, da die Gesamtkohorte zu diesem Zeitpunkt - bevor insbesondere die Schulabgänger*innen nach Klasse 10 nicht mehr im Rahmen von KESS erfasst wurden - die Referenzgröße darstellte.

Zusammenhängend damit sind weitere Einschränkungen verbunden, da der Bildungsweg nach Klasse 10 nicht für die gesamte Schülerkohorte erfasst wurde, sondern für größere Teile der Stichprobe rekonstruiert werden musste. In diesen Fällen wurde zunächst angenommen, dass keine Studienintention vorliegt. Die Studienintention auf Basis der gesamten Ausgangskohorte (inklusive Analysestrategie: Abgänger*innen nach der 10. Jahrgangsstufe und während der Oberstufe) wird hierbei insofern sehr konservativ interpretiert, da für einige Schüler*innen möglicherweise auch eine Studienintention vorliegen könnte (z.B. für die Wechsler*innen in andere Bundesländer und für krankheitsbedingt verhinderte Schüler*innen). Mit der exklusiven Analysestrategie, in der neben den an der KESS-Abschlusserhebung in der Oberstufe teilnehmenden Abiturient*innen nur die Abgänger*innen nach der Sekundarstufe I (Studienintention =0) zusätzlich einbezogen wurden, haben wir die Befunde auf Robustheit geprüft. Die resultierenden, nur leicht geringer ausfallenden sekundären Effekte stellen sozusagen die untere Grenze für das Ausmaß direkter Effekte der sozialen Herkunft am Übergang in die Hochschule für die Ausgangskohorte dar. Die inklusive Strategie konnte in weiterführenden (hier nicht im Detail berichteten) Analysen zudem durch multiple Imputation der Studienintention abgesichert werden. Auf der Datengrundlage der Gesamtkohorte (KESS 10/11) wurde dabei weiterhin angenommen, dass Schulabgänger*innen nach der 10. Jahrgangstufe keine Studienintention aufweisen, fehlende Informationen unter den Übergänger*innen in die Oberstufe (Ausscheiden aus KESS oder fehlende Werte zu KESS 12/13) wurden multipel imputiert (Methodik vgl. Abschn. 4.3). Die relativen Anteile sekundärer Effekte fielen auch mit diesem Vorgehen weitgehend ähnlich aus (HISEI: bis zu 3 Prozentpunkte höher, Bildungshintergrund: einen Prozentpunkt geringer), sodass mit den Ergebnissen dieser Studie auch die obere Grenze für das Ausmaß direkter Effekte der sozialen Herkunft am Übergang in die Hochschule ermittelt werden konnte. Zu bemerken ist allerdings, dass sich auf Basis der imputierten Daten größere totale Herkunftseffekte zeigten, die sogar die Effekte am Übergang in die Oberstufe leicht überstiegen. Auch wenn die Befunde insgesamt nicht auf deutliche Verschiebungen in den Ergebnismustern hinweisen, ist zu betonen, dass die hier vorgenommenen Schätzungen der Studienintention mit einem gewissen Vorbehalt zu interpretieren sind; insbesondere deshalb, weil sie auf den Leistungsdaten zum Zeitpunkt der 10./11. Jahrgangsstufe beruhen.

Generell ist als mögliche Einschränkung weiterhin zu berücksichtigen, dass Schüler*innen, die zum Ende ihrer Schulkarriere zunächst keine Studienintention äußern, ihre Pläne im weiteren Verlauf korrigieren können - wenngleich sich in der Forschung gezeigt hat, dass die am Ende der Oberstufe geäußerte Studienintention insgesamt eine gute Approximation für die spätere Aufnahme eines Studiums darstellt (Maaz 2006). 
Der Fokus dieser Studie lag auf der jeweils punktuellen querschnittlichen Effektdekomposition sozialer Herkunftseffekte. Zukünftige Untersuchungen sollten darüberhinausgehend längsschnittliche Untersuchungsperspektiven aufgreifen, um frühzeitig auftretende Ursachen späterer Bildungsentscheidungen und daraus resultierende Bildungsdisparitäten zu untersuchen. Hier sollten primäre und sekundäre Effekte nicht nur für getroffene Übergangsentscheidungen, sondern auch auf tatsächlich erreichte Bildungsabschlüsse hin untersucht werden. Dabei wäre aus schulstruktureller Perspektive auch von Interesse, inwieweit sich eine Transformation sekundärer Effekte an frühen Übergangsschwellen in primäre Effekte vollzieht, indem Entscheidungen für oder gegen höhere Bildungsgänge durch differenzielle Leistungsentwicklungen in getrennten Schulformen (Baumert et al. 2006) lernmilieubedingt in primäre Effekte umgewandelt werden.

Soziale Ungleichheiten beim Wechsel auf das Gymnasium beruhen nach Ergebnissen dieser Studie zu etwas mehr als der Hälfte auf sozialen Leistungsdisparitäten. Im Umkehrschluss lässt sich daraus ableiten, dass sich auch der „Elternwille“ für die Wahl der weiterführenden Schule bei nicht bindender Übergangsempfehlung (vgl. Gresch et al. 2010), in Form des sekundären Effekts, im Übergangsverhalten niederschlägt. Wie sich außerdem gezeigt hat, scheint vor allem der höchste familiäre Schulabschluss insbesondere zu späteren Übergangsschwellen ein entscheidendes Kriterium für eine Dominanz sekundärer Effekte zu sein. Zugleich haben sich größere absolute Herkunftseffekte des familiären Bildungshintergrunds im Vergleich zum sozioökonomischen Status gezeigt. Aufgrund der verschiedenen Maße sind die Effekte insbesondere absolut betrachtet allerdings nur eingeschränkt vergleichbar. Hier zeichnet sich ein Desiderat für zukünftige Forschungsarbeiten ab: Es gilt zu klären, wie die soziale Herkunft durch verschiedene Indikatoren für spezifische Effekte (vgl. Blossfeld 2018), aber auch in ihrer Vorhersagekraft für Bildungsungleichheiten bestmöglich abgebildet werden kann. Wie Meulemann und Relikowski (2016, S. 448f.) unter anderem mit Bezug auf Becker (2006) annehmen, stehen Bildungschancen in engerem Zusammenhang mit dem elterlichen Bildungsniveau als mit dem sozioökonomischen Status. Dies stützen auch die vorliegenden Ergebnisse. Gerade mit Blick auf Bildungsentscheidungen ließe sich daraus ableiten, dass etwa das Wissen innerhalb von Familien über höhere Bildungsgänge und damit verknüpfte Bildungsrenditen auch vom eigenen Bildungshintergrund abhängt. Einer Reduzierung von Bildungsungleichheiten ließe sich folglich mit vermehrter Information an Schulen über die Chancen weiterführender Bildung begegnen (vgl. Peter et al. 2016).

Danksagung Wir danken Karin Guill, Anna Bachsleitner und Gerrit Hasche für hilfreiche Kommentare und die Unterstützung bei der Manuskripterstellung.

Förderung Das Projekt „Primäre und sekundäre Herkunftseffekte an unterschiedlichen Gelenkstellen des Bildungssystems über den gesamten Verlauf der Sekundarstufe“ wird gefördert durch die Deutsche Forschungsgemeinschaft (DFG) (Projektnummer: 316563965).

Funding Open Access funding enabled and organized by Projekt DEAL.

Open Access Dieser Artikel wird unter der Creative Commons Namensnennung 4.0 International Lizenz veröffentlicht, welche die Nutzung, Vervielfältigung, Bearbeitung, Verbreitung und Wiedergabe in jeglichem Medium und Format erlaubt, sofern Sie den/die ursprünglichen Autor(en) und die Quelle ord- 
nungsgemäß nennen, einen Link zur Creative Commons Lizenz beifügen und angeben, ob Änderungen vorgenommen wurden.

Die in diesem Artikel enthaltenen Bilder und sonstiges Drittmaterial unterliegen ebenfalls der genannten Creative Commons Lizenz, sofern sich aus der Abbildungslegende nichts anderes ergibt. Sofern das betreffende Material nicht unter der genannten Creative Commons Lizenz steht und die betreffende Handlung nicht nach gesetzlichen Vorschriften erlaubt ist, ist für die oben aufgeführten Weiterverwendungen des Materials die Einwilligung des jeweiligen Rechteinhabers einzuholen.

Weitere Details zur Lizenz entnehmen Sie bitte der Lizenzinformation auf http://creativecommons.org/ licenses/by/4.0/deed.de.

\section{Literatur}

Baumert, J., Dumont, H., Becker, M., Neumann, M., Bachsleitner, A., Köller, O., \& Maaz, K. (2018). Soziokulturelle und geschlechtsspezifische Selektivität von Übergangsberechtigungen für die gymnasiale Oberstufe in mehr- und zweigliedrigen Schulsystemen. Kölner Zeitschrift für Soziologie und Sozialpsychologie, 70(4), 593-628.

Baumert, J., Stanat, P., \& Watermann, R. (2006). Schulstruktur und die Entstehung differenzieller Lernund Entwicklungsmilieus. In J. Baumert, P. Stanat \& R. Watermann (Hrsg.), Herkunftsbedingte Disparitäten im Bildungswesen: Differenzielle Bildungsprozesse und Probleme der Verteilungsgerechtigkeit (S. 95-188). Wiesbaden: VS.

Becker, R. (2003). Educational expansion and persistent inequalities of education: Utilizing subjective expected utility theory to explain increasing participation rates in upper secondary school in the Federal Republic of Germany. European Sociological Review, 19(1), 1-24.

Becker, R. (2006). Dauerhafte Bildungsungleichheiten als unerwartete Folge der Bildungsexpansion? In A. Hadjar \& R. Becker (Hrsg.), Die Bildungsexpansion: Erwartete und unerwartete Folgen (S. 27-61). Wiesbaden: VS.

Becker, R. (2009). Wie können „,bildungsferne“ Gruppen für ein Hochschulstudium gewonnen werden? Eine empirische Simulation mit Implikationen für die Steuerung des Bildungswesens. Kölner Zeitschrift für Soziologie und Sozialpsychologie, 61(4), 563-593.

Becker, R., \& Lauterbach, W. (2016). Bildung als Privileg - Ursachen, Mechanismen, Prozesse und Wirkungen. In R. Becker \& W. Lauterbach (Hrsg.), Bildung als Privileg. Erklärungen und Befunde zu den Ursachen der Bildungsungleichheit (5. Aufl., S. 3-53). Wiesbaden: Springer VS.

Blossfeld, P.N. (2018). Social background and between-track mobility in the general education system in West Germany and in East Germany after German unification. Zeitschrift für Soziologie, 47(4), 255-269.

Blossfeld, H.-P., \& Shavit, Y. (1993). Dauerhafte Ungleichheiten. Zur Veränderung des Einflusses der sozialen Herkunft auf die Bildungschancen in dreizehn industrialisierten Ländern. Zeitschrift für Pädagogik, 39(1), 25-52.

Bos, W., \& Pietsch, M. (Hrsg.). (2006). KESS 4 - Kompetenzen und Einstellungen von Schülerinnen und Schülern an Hamburger Schulen am Ende der Jahrgangsstufe 4 in Hamburger Grundschulen. Münster: Waxmann.

Boudon, R. (1974). Education, opportunity, and social inequality: changing prospects in western society. New York: John Wiley \& Sons.

van Buuren, S., \& Groothuis-Oudshoorn, K. (2011). MICE: multivariate imputation by chained equations in R. Journal of Statistical Software, 45(3), 1-67.

Ditton, H., \& Krüsken, J. (2006). Der Übergang von der Grundschule in die Sekundarstufe I. Zeitschrift für Erziehungswissenschaft, 9(3), 348-372.

Dumont, H., Maaz, K., Neumann, M., \& Becker, M. (2014). Soziale Ungleichheiten beim Übergang von der Grundschule in die Sekundarstufe I: Theorie, Forschungsstand, Interventions- und Fördermöglichkeiten. In K. Maaz, M. Neumann \& J. Baumert (Hrsg.), Herkunft und Bildungserfolg von der frühen Kindheit bis ins Erwachsenenalter: Forschungsstand und Interventionsmöglichkeiten aus interdisziplinärer Perspektive (Zeitschrift für Erziehungswissenschaft: Sonderheft 5, S. 141-165). Wiesbaden: Springer VS. 
Erikson, R., Goldthorpe, J.H., Jackson, M., Yaish, M., \& Cox, D. R. (2005). On class differentials in educational attainment. Proceedings of the National Academy of Sciences of the United States of America, 102(27), 9730-9733.

Esser, H. (1999). Situationslogik und Handeln (Soziologie. Spezielle Grundlagen, Bd. 1). Frankfurt a. M.: Campus.

Esser, H. (2016). Bildungssysteme und ethnische Bildungsungleichheiten. In C. Diehl, C. Hunkler \& C. Kristen (Hrsg.), Ethnische Ungleichheiten im Bildungsverlauf: Mechanismen, Befunde, Debatten (S. 331-396). Wiesbaden: Springer.

Feddermann, M., Guill, K., List, M. K., Matthießen, R., Ömeroğulları, M., Stallasch, S.E., Köller, O., \& Nagy, G. (2019). KESS - Skalierung der Leistungstests. Kiel: Leibniz-Institut für die Pädagogik der Naturwissenschaften und Mathematik (IPN).

Ganzeboom, H. B. G., de Graaf, P. M., \& Treiman, D. J. (1992). A standard international socio-economic index of occupational-status. Social Science Research, 21(1), 1-56.

Graham, J. W. (2009). Missing data analysis: making it work in the real world. Annual Review in Psycho$\log y$, , 60, 549-576.

Gresch, C., Baumert, J., \& Maaz, K. (2010). Empfehlungsstatus, Übergangsempfehlung und der Wechsel in die Sekundarstufe I. Bildungsentscheidungen und soziale Ungleichheit. In K. Maaz, J. Baumert, C. Gresch \& N. McElvany (Hrsg.), Der Übergang von der Grundschule in die weiterführende Schule. Leistungsgerechtigkeit und regionale, soziale und ethnisch-kulturelle Disparitäten (S. 201-228). Bonn: Bundesministerium für Bildung und Forschung, Referat Bildungsforschung.

Helbig, M., \& Morar, T. (2017). Warum Lehrkräfte sozial ungleich bewerten. Ein Plädoyer für die Etablierung tertiärer Herkunftseffekte im werterwartungstheoretischen Standardmodell der Bildungsforschung. Berlin: Wissenschaftszentrum Berlin für Sozialforschung, Projektgruppe der Präsidentin.

Hillmert, S., \& Jacob, M. (2005). Institutionelle Strukturierung und inter-individuelle Variation. Die Entwicklung herkunftsbezogener Ungleichheiten im Bildungsverlauf. Kölner Zeitschrift für Soziologie und Sozialpsychologie, 57(3), 414-442.

Karlson, K. B., \& Holm, A. (2011). Decomposing primary and secondary effects: a new decomposition method. Research in Social Stratification and Mobility, 29(2), 221-237.

Karlson, K.B., Holm, A., \& Breen, R. (2012). Comparing regression coefficients between same-sample nested models using logit and probit: a new method. Sociological Methodology, 42, 286-313.

Lehberger, R., \& d Lorent, H.-P. (2012). Schulen in Hamburg. Ein Führer durch Aufbau und Geschichte des Hamburger Schulwesens. Hamburg: Brunswiker + Reuter.

Lüdtke, O., \& Robitzsch, A. (2010). Umgang mit fehlenden Daten in der empirischen Bildungsforschung. In S. Maschke \& L. Stecher (Hrsg.), Enzyklopädie Erziehungswissenschaft Online. Fachgebiet Methoden der empirischen erziehungswissenschaftichen Forschung, Quantitative Forschungsmethoden (S. 1-42). Weinheim: Juventa.

Maaz, K. (2006). Soziale Herkunft und Hochschulzugang: Effekte institutioneller Öffnung im Bildungssystem. Wiesbaden: VS.

Maaz, K., \& Nagy, G. (2009). Der Übergang von der Grundschule in die weiterführenden Schulen des Sekundarschulsystems: Definition, Spezifikation und Quantifizierung primärer und sekundärer Herkunftseffekte. In J. Baumert, K. Maaz \& U. Trautwein (Hrsg.), Bildungsentscheidungen (Zeitschrift für Erziehungswissenschaft: Sonderheft 12, S. 153-182). Wiesbaden: VS.

Maaz, K., Bachsleitner, A., Baumert, J., Dumont, H., Neumann, M., Radmann, S., \& Becker, M. (2017). Soziokulturelle Disparitäten des Bildungserfolgs vor und nach der Schulstrukturreform in Berlin. In M. Neumann, M. Becker, J. Baumert, K. Maaz \& O. Köller (Hrsg.), Zweigliedrigkeit im deutschen Schulsystem. Potenziale und Herausforderungen in Berlin (S. 361-424). Münster: Waxmann.

Maaz, K., Baumert, J., \& Trautwein, U. (2009). Genese sozialer Ungleichheit im institutionellen Kontext der Schule: Wo entsteht und vergrößert sich soziale Ungleichheit? In J. Baumert, K. Maaz \& U. Trautwein (Hrsg.), Bildungsentscheidungen (Zeitschrift für Erziehungswissenschaft: Sonderheft 12, S. 11-46). Wiesbaden: VS.

Mare, R. D. (1980). Social background and school continuation decisions. Journal of the American Statistical Association, 75, 295-305.

Meulemann, H., \& Relikowski, I. (2016). Chancengleichheit und Leistung im sozialen Wandel. Primäre und sekundäre Effekte der sozialen Herkunft am Gymnasialübergang in Hessen 1969 und 2007. Zeitschrift für Erziehungswissenschaft, 19(2), 443-462.

Müller-Benedict, V. (2007). Wodurch kann die soziale Ungleichheit des Schulerfolgs am stärksten verringert werden? Kölner Zeitschrift für Soziologie und Sozialpsychologie, 59(4), 615-639. 
Neugebauer, M. (2010). Bildungsungleichheit auf Grundschulempfehlung beim Übergang auf das Gymnasium: Eine Dekomposition primärer und sekundärer Herkunftseffekte. Zeitschrift für Soziologie, 39(3), 202-214.

Neugebauer, M., \& Schindler, S. (2012). Early transitions and tertiary enrolment: the cumulative impact of primary and secondary effects on entering university in Germany. Acta Sociologica, 55(1), 19-36.

Neugebauer, M., Reimer, D., Schindler, S., \& Stocké, V. (2013). Inequality in transition to secondary school and tertiary education in Germany. In M. Jackson (Hrsg.), Determined to succeed? Performance versus choice in educational attainment (S. 56-88). Stanford: Stanford University Press.

Neumann, M., Becker, M., \& Maaz, K. (2014). Soziale Ungleichheiten in der Kompetenzentwicklung in der Grundschule und der Sekundarstufe I. In K. Maaz, M. Neumann \& J. Baumert (Hrsg.), Herkunft und Bildungserfolg von der frühen Kindheit bis ins Erwachsenenalter: Forschungsstand und Interventionsmöglichkeiten aus interdisziplinärer Perspektive (Zeitschrift für Erziehungswissenschaft: Sonderheft 5, S. 167-203). Wiesbaden: Springer VS.

Peter, F.H., Zambre, V., \& Spieß, C. K. (2016). Die Bereitstellung von Informationen beeinflusst die Bildungsentscheidung von Abiturientinnen und Abiturienten. Zeitschrift für Beratung und Studium, 11(3), 66-72.

Pietsch, M. (2007). Schulformwahl in Hamburger Schülerfamilien und die Konsequenzen für die Sekundarstufe I. In W. Bos, C. Gröhlich \& M. Pietsch (Hrsg.), KESS 4 - Lehr- und Lernbedingungen in Hamburger Grundschulen (S. 127-165). Münster: Waxmann.

R Core Team (2017). R: A language and environment for statistical computing, Wien. https://www.Rproject.org/. Zugegriffen: 30. Juli 2019.

Rubin, D.B. (1987). Multiple imputation for nonresponse in surveys. New York: Wiley.

Schindler, S., \& Lörz, M. (2012). Mechanism of social inequality development: Primary and secondary effects in the transition to tertiary education between 1976 and 2005. European Sociological Review, 28(5), 647-660.

Schindler, S., \& Reimer, D. (2010). Primäre und sekundäre Effekte der sozialen Herkunft beim Übergang in die Hochschulbildung. Kölner Zeitschrift für Soziologie und Sozialpsychologie, 62(4), 623-653.

Schneider, H., Franke, B., Woisch, A., \& Spangenberg, H. (2017). Erwerb der Hochschulreife und nachschulische Übergänge von Studienberechtigten. Studienberechtigte 2015 ein halbes Jahr vor und ein halbes Jahr nach Schulabschluss (Forum Hochschule 412017). Hannover: DZHW.

Stocké, V. (2007a). Explaining educational decision and effects of families' social class position: An empirical test of the Breen-Goldthorpe model of educational attainment. European Sociological Review, 23(4), 505-519.

Stocké, V. (2007b). Strength, sources, and temporal development of primary effects of families' social status on secondary school choice (Working Paper. Sonderforschungsbereich 504). Mannheim: Universität Mannheim.

Stubbe, T.C. (2009). Bildungsentscheidungen in der Sekundarstufe I - Sekundäre Herkunftseffekte an Hamburger Schulen. Zeitschrift für Soziologie der Erziehung und Sozialisation, 29(4), 419-436.

Trautwein, U., Nagy, G., \& Maaz, K. (2011). Soziale Disparitäten und die Öffnung des Sekundarschulsystems: Eine Studie zum Übergang von der Realschule in die gymnasiale Oberstufe. Zeitschrift für Erziehungswissenschaft, 14(3), 445-463.

Vieluf, U., Ivanov, S., \& Nikolova, R. (Hrsg.). (2011). KESS 10/11 - Kompetenzen und Einstellungen von Schülerinnen und Schülern an Hamburger Schulen am Ende der Sekundarstufe I und zu Beginn der gymnasialen Oberstufe. Münster: Waxmann.

Vieluf, U., Ivanov, S., \& Nikolova, R. (2014). KESS 12/13 - Kompetenzen und Einstellungen von Schülerinnen und Schülern an Hamburger Schulen am Ende der Sekundarstufe I und zu Beginn der gymnasialen Oberstufe. Hamburg. https://bildungsserver.hamburg.de/contentblob/4396048/ 6b49c68061321ae400aaa4f7250ebe9f/data/kess12-13.pdf. Zugegriffen: 26. Juni 2019.

Watermann, R., Daniel, A., \& Maaz, K. (2014). Primäre und sekundäre Disparitäten des Hochschulzugangs: Erklärungsmodelle, Datengrundlagen und Entwicklungen. In K. Maaz, M. Neumann \& J. Baumert (Hrsg.), Herkunft und Bildungserfolg von der frühen Kindheit bis ins Erwachsenenalter: Forschungsstand und Interventionsmöglichkeiten aus interdisziplinärer Perspektive (Zeitschrift für Erziehungswissenschaft: Sonderheft 5, S. 233-261). Wiesbaden: Springer VS. 